\title{
Rumor spreading of a SEIR model in complex social networks with hesitating mechanism
}

\author{
Xiongding $\mathrm{Liu}^{1,2} \mathbb{D}$, Tao Li $\mathrm{Li}^{1,2^{*}}$ and Mi Tian ${ }^{3}$
}

\section{"Correspondence:}

taohust2008@163.com

National Demonstration Center for

Experimental Electrical and

Electronic Education, Yangtze

University, Jingzhou, P.R. China

${ }^{2}$ School of Electronics and

Information, Yangtze University,

Jingzhou, P.R. China

Full list of author information is

available at the end of the article

\begin{abstract}
In the process of rumor spreading, controlling and killing rumor problem is of great importance on social networks. In this paper, we present a new SEIR

(susceptible-exposed-infected-removed) rumor spreading model with hesitating mechanism. By using mean-field theory, the equilibrium of the model and the basic reproduction number $R_{0}$ are obtained. The global stability of the rumor-free equilibrium and the permanence are proved in detail, and the global attractivity of the rumor-prevailing equilibrium is proved by using a monotone iterative technique. Furthermore, the modified model with feedback mechanism on social networks is introduced. The feedback mechanism cannot change the basic reproductive number but it can reduce the continuous level and the spread of rumor. Numerical simulations confirm the analytical results.
\end{abstract}

Keywords: Rumor spreading; Social networks; Hesitating mechanism; Equilibrium; Global attractivity

\section{Introduction}

Rumors are usually defined as unconfirmed elaborations or annotations of public things, events, or issues that are fabricated and propagated through various means [1, 2], which can cause unnecessary public anxiety and economic loss to affected countries [3-5]. What's worse, with the development of network communications, rumor has propagated more quickly and widely [6-8]. Therefore, the study of rumor spreading model is of dramatic importance, which plays a significant role in managing and controlling the spread of rumor.

Rumor spreading in complex social networks has recently attracted an increasing amount of attention from researchers [9-12]. They describe the dynamics of the models by deriving corresponding mean-field equations to formulate the characteristics and analyze the critical threshold of rumor spreading in the complex networks. In 1965, Daley and Kendall put forward the first classical $D K$ rumor spreading model [13]. Some researchers applied a mathematical model to study the rumors and developed another classical model [14-17]. Early classical representations of rumor spreading dynamics assumed that all individuals have the homogeneous probability of connection [18-20]. Obviously, these simple models can not completely reflect the realistic feature of the spread of rumor, which is subsequently extended in ways to make them more realistic in recent years.

(c) The Author(s) 2018. This article is distributed under the terms of the Creative Commons Attribution 4.0 International License (http://creativecommons.org/licenses/by/4.0/), which permits unrestricted use, distribution, and reproduction in any medium, provided you give appropriate credit to the original author(s) and the source, provide a link to the Creative Commons license, and indicate if changes were made. 
Such extensions are combining with spreading mode that the online networks platform could affect the topology of rumor spreading network. Another direction to research rumor spreading model is to focus on the complex topology of social interactions. In the field of social network, the scale-free property is the fundamental characteristic, and the nodes stand for individuals and the edges stand for various interactions of people. Therefore, it makes more sense to study spreading dynamics on scale-free networks [21-26]. For further understanding of the rumor spreading dynamic in the real world, the counterattack and self-resistance mechanisms, trust mechanism, latent mechanism, rumor-killer mechanism, and information pushing mechanism [27-33] have been taken into account. These models more accurately reflect the spread of rumors and also give a lot of control strategies for rumors.

Undeniably, the influences of hesitating mechanisms also play an important role in the process of rumor spreading. Recently, in order to investigate the influence of heterogeneity of the underlying complex networks and hesitating mechanisms on rumor spreading, Liu et al. proposed a SEIR rumor propagation model on the heterogeneous networks [34]. They found that the spreading threshold significantly depends on the topology of the complex networks and analyzed globally dynamic behaviors of the rumor. Wan et al. presented a SEIR rumor spreading model with demographics on scale-free networks [35]. They proved the global asymptotic stability of rumor-free equilibrium and the permanence of the rumor, but the simulation of the experiment was not given, and the direct immunization of rumor spreading was not considered. However, in real life, some wise people or functional departments which realize the information is rumor will take appropriate measures to prevent its continued spreading in the early days when rumors begin to spread, i.e., direct immunization [36]. Furthermore, the initiative response of individual is not considered when rumors spreading prevail, i.e., feedback mechanism. In fact, once people know the harm of rumor, they will question and further verify the rumor, and thus reduce the trust and the spread of rumor. Obviously, the feedback mechanism can change the network topology structure [37, 38]. In this paper, considering the direct immunization, we focus on a SEIR rumor model in social networks, we comprehensively prove the permanence of the rumor spreading in detail. Meanwhile, the modified model with feedback mechanism in social networks is introduced.

The rest of the paper is organized as follows: in Sect. 2, we present a new SEIR model in social networks. In Sect. 3, the dynamical behaviors of rumor spreading are analyzed in detail. Section 4 gives theoretical analysis to the stability of the equilibrium. In Sect. 5, the modified model feedback mechanism in social networks is introduced. In Sect. 6, numerical simulations are given to illustrate the main results. Finally, the conclusions are given in Sect. 7.

\section{Model formulation}

In this paper, we consider the whole population $N_{k}(t)$ to be located in a relevant social network. Users can be regarded as nodes and direct relationships between users can be considered as edges. Our model is based on dividing the whole population into four states: $S, E, I$, and $R$. At each time step, each individual adopts one of four states $S, E, I$, or $R$, which respectively stand for the people who have never contacted with rumor (susceptible), the people who have been infected, the people who have been in hesitate state and do not spread rumor (exposed), the people who are actively spreading it (infected), and the 
Figure 1 Transfer diagram for rumor spreading model

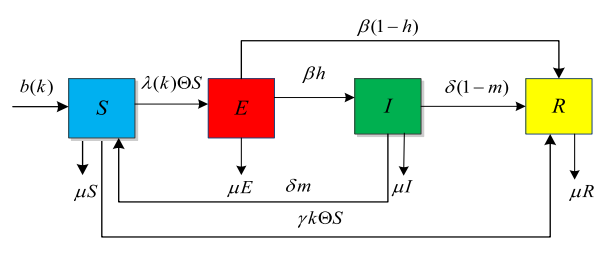

people who have known the rumor but never believed and spread it (removed). Taking the heterogeneity induced by the presence of nodes with different connectivities into account, we let $S_{k}(t), E_{k}(t), I_{k}(t)$, and $R_{k}(t)$ be the relative densities of susceptible, infected, and recovered nodes of degree $k$ at time $t$, respectively. The process of SEIR rumor spreading is shown in Fig. 1.

In the process of rumor spreading, the spreading among these four states is governed by the following rules: we assume that $\lambda(k)>0$ is the degree-dependent infected rate, and it denotes the acceptability of $k$ degree nodes for rumors. The susceptible nodes know the rumor but do not trust at rate $\gamma$ if it connects to an infected one, the exposed node is infected with probability $\beta, h$ represents the attractiveness of rumor itself on the complex social network, and the corresponding repellant of rumor is $1-h$. The fuzziness of rumor is $m(0 \leq m \leq 1)$ and the corresponding clarity of rumor is $1-m$. The forgetting rate is $\delta$. Due to trust and interest in the rumor, the exposed node becomes an infected node with probability $\beta h$, and with probability $\beta(1-h)$, it becomes a recover node. Because of the fuzziness of the rumor, the parameters $\delta m$ and $\delta(1-m)$ represent that the infected node has become susceptible and removed, respectively. The degree-dependent parameter $b(k)>0$ represents the number of newly immigrated individuals with degree $k$ per unit time, and each newly immigrated individual is susceptible, the reconnection of these nodes follows the above propagation rules. This type of rewiring preserves the network mean degree (the total number of links remains constant) but changes the mean degree of susceptible and infected nodes [39-41]. The emigration is proportional to the node size with probability $\mu$. The parameters are all nonnegative. The model can be described by the following system of ordinary differential equations:

$$
\left\{\begin{array}{l}
\frac{d S_{k}(t)}{d t}=b(k)-\lambda(k) \Theta(t) S_{k}(t)-\gamma k \Theta(t) S_{k}(t)-\mu S_{k}(t)+\delta m I_{k}(t), \\
\frac{d E_{k}(t)}{d t}=\lambda(k) \Theta(t) S_{k}(t)-\mu E_{k}(t)-\beta E_{k}(t), \\
\frac{d I_{k}(t)}{d t}=\beta h E_{k}(t)-\delta I_{k}(t)-\mu I_{k}(t), \\
\frac{d R_{k}(t)}{d t}=\gamma k \Theta(t) S_{k}(t)+\beta(1-h) E_{k}(t)+\delta(1-m) I_{k}(t)-\mu R_{k}(t),
\end{array}\right.
$$

$\Theta(t)$ denotes the probability of ignoramus contacts with a sharer at time $t$, which satisfies the relation

$$
\Theta(t)=\sum_{i=1}^{n} \frac{\varphi(i)}{i} P(i \mid k) \frac{I_{i}(t)}{N_{i}(t)},
$$

where $P(i \mid k)$ denotes the conditional probability that a node with degree $k$ is connected to a node with degree $i$. Considering the uncorrelated network [42], in this paper, $P(i \mid k)=$ $i P(i) /\langle k\rangle$. Here, $P(k)$ is the probability that a randomly chosen node has degree $k$, thus $\sum_{k=1}^{n} P(k)=1,\langle k\rangle=\sum_{k=1}^{n} k P(k)$ denotes the average degree, and $\varphi(k)$ is a function that 
represents the infectivity of a node with degree $k$; the factor $1 / i$ stands for the probability that one of the infected neighbors of a node, with degree $i$, will contact this node at the present time step. Summing the four equations in (2.1), we can obtain

$$
\begin{aligned}
& \frac{d N_{k}(t)}{d t}=b(k)-\mu N_{k}(t), \quad \text { then } N_{k}(t)=N_{k}(0) e^{-\mu t}+\frac{b(k)}{\mu}\left(1-e^{-\mu t}\right), \\
& \lim _{t \rightarrow \infty} N_{k}(t)=\frac{b(k)}{\mu} .
\end{aligned}
$$

We suppose that $N_{k}(0)=S_{k}(0)+E_{k}(0)+I_{k}(0)+R_{k}(0)=\frac{b(k)}{\mu}$ in order to have a population of constant size, namely $S_{k}(t)+E_{k}(t)+I_{k}(t)+R_{k}(t)=N_{k}(t) \equiv \eta_{k}$, where $\eta_{k}=b(k) / \mu$. So we can obtain

$$
\Theta(t)=\frac{1}{\langle k\rangle} \sum_{i=1}^{n} \frac{\varphi(k)}{\eta_{k}} P(k) I_{k}(t)
$$

Furthermore, $S(t)=\sum_{k=1}^{n} P(k) S_{k}(t), E(t)=\sum_{k=1}^{n} P(k) E_{k}(t), I(t)=\sum_{k=1}^{n} P(k) I_{k}(t), R(t)=$ $\sum_{k=1}^{n} P(k) R_{k}(t)$ are the average densities of the four individual states, respectively. The initial conditions for system can be given as follows: $S_{k}(0)=\eta_{k}-E_{k}(0)-I_{k}(0)-R_{k}(0)>$ $0, E_{k}(0) \geq 0, I_{k}(0) \geq 0, R_{k}(0) \geq 0$, and $\Theta(0)>0$.

\section{The basic reproduction number and existence of equilibriums}

In this section, we present an analytic solution to the deterministic equations describing the dynamics of the SEIR rumor spreading process in social networks.

Theorem 1 Consider system (2.1), let $R_{0}=\frac{\langle\varphi(k) \lambda(k)\rangle \beta h}{\langle k\rangle(\delta+\mu)(\beta+\mu)}$. There always exists a rumorfree equilibrium $E_{0}(b(k) / \mu, 0,0,0)$ when $R_{0}<1$. When $R_{0}>1$, system (2.1) has a rumorprevailing equilibrium $E_{+}\left(S_{k}^{*}, E_{k}^{*}, I_{k}^{*}, R_{k}^{*}\right)$.

Proof To get the rumor-prevailing equilibrium solution $E_{+}\left(S_{k}^{*}, E_{k}^{*}, I_{k}^{*}, R_{k}^{*}\right)$, we need to make the right-hand side of the system equal to zero, it should satisfy

$$
\left\{\begin{array}{l}
b(k)+\delta m I_{k}^{*}-\mu S_{k}^{*}-\lambda(k) \Theta^{*} S_{k}^{*}-\gamma k \Theta^{*} S_{k}^{*}=0 \\
\lambda(k) \Theta^{*} S_{k}^{*}-\mu E_{k}^{*}-\beta E_{k}^{*}=0 \\
\beta h E_{k}^{*}-\delta I_{k}^{*}-\mu I_{k}^{*}=0 \\
\gamma k \Theta^{*} S_{k}^{*}+\delta(1-m) I_{k}^{*}+\beta(1-h) E_{k}^{*}-\mu R_{k}^{*}=0 \\
S_{k}^{*}+E_{k}^{*}+I_{k}^{*}+R_{k}^{*}=\eta_{k}
\end{array}\right.
$$

where $\Theta^{*}=\frac{1}{\langle k\rangle} \sum_{i=1}^{n} \frac{\varphi(k)}{\eta_{k}} P(k) I_{k}^{*}(t)$. One has

$$
\left\{\begin{array}{l}
S_{k}^{*}=\frac{(\mu+\delta)(\beta+\mu)}{\lambda(k) \beta h \Theta^{*}} I_{k}^{*}, \\
E_{k}^{*}=\frac{\mu+\delta}{\beta h} I_{k}^{*}, \\
R_{k}^{*}=\frac{\gamma k(\beta+\mu)(\delta+\mu)+\beta \lambda(k)[(\delta+\mu)-h(\mu+\delta m)]}{\lambda(k) \mu \beta h} I_{k}^{*} .
\end{array}\right.
$$


Considering the following normalization condition $S_{k}(t)+E_{k}(t)+I_{k}(t)+R_{k}(t)=\eta_{k}$ for all $k$, we obtain

$$
I_{k}^{*}=\frac{\mu \lambda(k) \Theta^{*} \beta h \eta_{k}}{\left(\mu+\gamma k \Theta^{*}\right)(\beta+\mu)(\delta+\mu)+\lambda(k) \Theta^{*}((\beta+\mu)(\delta+\mu)-\beta h \delta m)} .
$$

Inserting into (2.2), we obtain that

$$
\Theta^{*}=\frac{1}{\langle k\rangle} \sum_{i=1} \frac{\varphi(k)}{\eta_{k}} P(k) I_{k}^{*}
$$

For simplification, we substitute $\Theta$ for $\Theta^{*}$, then from (3.4) we have the self-consistency equation:

$$
\begin{aligned}
\Theta & =\frac{1}{\langle k\rangle} \sum_{i=1} \frac{\varphi(k)}{\eta_{k}} P(k) \frac{\lambda(k) \beta h \Theta \mu \eta_{k}}{(\mu+\gamma k \Theta)(\beta+\mu)(\delta+\mu)+\lambda(k) \Theta((\beta+\mu)(\delta+\mu)-\beta h \delta m)} \\
& =: f(\Theta) .
\end{aligned}
$$

Clearly, $\Theta=0$ is the solution of (3.5), i.e., $f(0) \leq 0$. The conditions under a nontrivial solution to (3.5) exist.

To ensure that the equation has a nontrivial solution $\Theta(\Theta \in(0,1))$, the following condition should be satisfied:

$$
\left.\frac{d f(\Theta)}{d \Theta}\right|_{\Theta=0}>1,
$$

which indicates that $\frac{\langle\varphi(k) \lambda(k)\rangle}{\langle k\rangle} \frac{\beta h}{(\delta+\mu)(\beta+\mu)}>1$, we can obtain the basic reproductive number

$$
R_{0}=\frac{\langle\varphi(k) \lambda(k)\rangle}{\langle k\rangle} \frac{\beta h}{(\delta+\mu)(\beta+\mu)} .
$$

So, a nontrivial solution exists if and only if $R_{0}>1$.

Inserting the nontrivial solution of (3.4) into Eq. (3.3), we can obtain $I_{k}^{*}$. By (3.2) and (3.3) we can easily get $0<S_{k}^{*}<\eta_{k}, 0<E_{k}^{*}<\eta_{k}, 0<I_{k}^{*}<\eta_{k}, 0<R_{k}^{*}<\eta_{k}$.

Thus, the equilibrium $E_{+}\left(S_{k}^{*}, E_{k}^{*}, I_{k}^{*}, R_{k}^{*}\right)$ is well-defined. Hence, when $R_{0}>1$, only one positive equilibrium $E_{+}\left(S_{k}^{*}, E_{k}^{*}, I_{k}^{*}, R_{k}^{*}\right)$ of system (2.1) exists. The proof is completed.

\section{Remark}

(1) The basic reproductive number $R_{0}$ is obtained by Eq. (3.7), it determines the existence of the endemic equilibrium. The details will be further verified in the next section. It can also be interpreted as the average number of secondary infections generated by an infected node during its infection time [43].

(2) The basic reproductive number $R_{0}$ depends on some parameters and the fluctuation of the degree distribution. Interestingly, $R_{0}$ has no correlation with the degree-dependent new immigrate $b(k)$ and the recovered rate $\gamma$. It seems that the attractive parameter $h$ and the infected rate $\beta$ have the same effects, because their increase will make $R_{0}$ increase. In Sect. 6 , their effects will be explored by the detailed numerical calculation. 
(3) If $\lambda(k)=\lambda k, \varphi(k)=k, b(k)=0$, and $\mu=0$, then system (2.1) becomes the network-based SEIRS model without demographics, and $R_{0}>1$ is simplified to $\lambda>\lambda_{c}$, where $\lambda_{c}=\frac{\delta\langle k\rangle}{\beta\left\langle k^{2}\right\rangle}$. It is clear that, in the infinite size network, the total number of nodes $N_{k}(t)$ grows to infinity, i.e., $N_{k}(t) \rightarrow \infty$, then $\left\langle k^{2}\right\rangle \rightarrow \infty$, thus the absence of the spreading threshold, i.e., $\lambda_{c} \rightarrow 0$, is observed.

\section{Stability analysis of the equilibrium}

Theorem 2 If $R_{0}<1$, the rumor-free equilibrium $E_{0}$ of system (2.1) is locally asymptotically stable, and it is unstable when $R_{0}>1$.

Proof For $S_{k}(t)+E_{k}(t)+I_{k}(t)+R_{k}(t)=\eta_{k}$, i.e., if the values of $E_{k}(t), I_{k}(t)$, and $R_{k}(t)$ are fixed, there is only one corresponding $S_{k}(t)$, therefore, it will be sufficient for us to discuss the last three equations of (2.1):

$$
\left\{\begin{array}{l}
\frac{d E_{k}(t)}{d t}=\lambda(k) \Theta(t) S_{k}(t)-\mu E_{k}(t)-\beta E_{k}(t), \\
\frac{d I_{k}(t)}{d t}=\beta h E_{k}(t)-\delta I_{k}(t)-\mu I_{k}(t), \\
\frac{d R_{k}(t)}{d t}=\gamma k \Theta(t) S_{k}(t)+\beta(1-h) E_{k}(t)+\delta(1-m) I_{k}(t)-\mu R_{k}(t),
\end{array}\right.
$$

where the Jacobian matrix of the rumor-free equilibrium $E_{0}$ of system (4.1) is

$$
\begin{aligned}
& J=\left[\begin{array}{cccc}
A_{11} & A_{12} & \cdots & A_{1 n} \\
A_{21} & A_{22} & \cdots & A_{2 n} \\
\vdots & \vdots & \vdots & \vdots \\
A_{n 1} & A_{n 2} & \cdots & A_{n n}
\end{array}\right]_{3 n \times 3 n} \quad \text { where } A_{i j}=\left(\begin{array}{ccc}
0 & \frac{\lambda(j) \varphi(j) P(j)}{\langle k\rangle} & 0 \\
0 & 0 & 0 \\
0 & 0 & 0
\end{array}\right) \\
& A_{n n}=\left(\begin{array}{ccc}
-(\beta+\mu) & -\frac{\lambda(j) \varphi(j) P(j)}{\langle k\rangle} & 0 \\
\beta h & -(\delta+\mu) & 0 \\
\beta(1-h) & \delta(1-m)-\frac{\lambda(j) \varphi(j) P(j)}{\langle k\rangle} & \mu
\end{array}\right) \quad(i, j=1,2, \ldots, n) .
\end{aligned}
$$

By using the mathematical induction method, the characteristic equation can be calculated as follows:

$$
(x+\mu)^{n}(x+\beta+\mu)^{n-1}(x+\delta+\mu)^{n-1}\left\{(x+\beta+\mu)(x+\delta+\mu)-\beta h \frac{\langle\lambda(k) \varphi(k)\rangle}{\langle k\rangle}\right\}=0,
$$

where $\sum_{i=1}^{n} \lambda(i) \varphi(i) P(i)=\lambda(1) \varphi(1) P(1)+\lambda(2) \varphi(2) P(2)+\cdots+\lambda(n) \varphi(n) P(n)=\langle\lambda(k) \varphi(k)\rangle$.

This equation has a negative root $-\mu$ with multiplicity $n$, a negative root $-\mu-\beta$ with multiplicity $n-1$, and a negative root $-\mu-\delta$ with multiplicity $n-1$. The stability of $E_{0}$ is only dependent on

$$
x^{2}+(\beta+\delta+2 \mu) x+(\delta+\mu)(\beta+\mu)-\beta h \frac{\langle\lambda(k) \varphi(k)\rangle}{\langle k\rangle}=0 \text {. }
$$

According to equation (4.3), if $R_{0}>1$, we can easily get $(\delta+\mu)(\beta+\mu)-\beta h \frac{\langle\lambda(k) \varphi(k)\rangle}{\langle k\rangle}>0$, that is, $x<0$. If $x=R_{0}<1$, then $x<0$, and if $x=R_{0}>1$, then $x>0$. Thus, $E_{0}$ is locally asymptotically stable when $R_{0}>1$. The proof is completed. 
In the following, we consider global asymptotic stability of $E_{0}$ and the global attractivity of $E^{*}$, which is one of the most important topics in the study of rumor spreading.

Lemma 4.1 ([44]) If $a>0, b>0$, and $\frac{d x(t)}{d t} \geq b-a x$, when $t \geq 0$ and $x(0) \geq 0$, we have $\lim _{t \rightarrow \infty} \sup x(t) \leq \frac{b}{a}$, if $a>0, b>0$, and $\frac{d x(t)}{d t} \leq b-a x$, when $t \geq 0$ and $x(0) \geq 0$, we have $\lim _{t \rightarrow \infty} \sup x(t) \leq \frac{b}{a}$.

Theorem 3 If $R_{0}<1$, the rumor-free equilibrium $E_{0}$ of system (2.1) is globally asymptotically stable.

Proof First, we define a Lyapunov function $V(t)$ as follows:

$$
V(t)=\sum_{k} \frac{\varphi(k)}{\eta_{k}} P(k) E_{k}(t)+\frac{(\beta+\mu)}{\beta h} \sum_{k} \frac{\varphi(k)}{\eta_{k}} P(k) I_{k}(t) .
$$

Then, according to a calculation of the derivative of $V(t)$ along the solution of system (2.1), we get

$$
\begin{aligned}
\dot{V}(t)= & \sum \frac{\varphi(k)}{\eta_{k}} P(k)\left(\lambda(k) \Theta(t) S_{k}(t)-\mu E_{k}(t)-\beta E_{k}(t)\right) \\
& +\sum \frac{(\beta+\mu)}{\beta h} \frac{\varphi(k)}{\eta_{k}}\left(\beta h E_{k}(t)-\delta I_{k}(t)-\mu I_{k}(t)\right) \\
= & \sum \frac{\varphi(k)}{\eta_{k}} P(k) \lambda(k) \Theta(t) S_{k}(t)+\sum \frac{(\beta+\mu)}{\beta h} \frac{\varphi(k)}{\eta_{k}}\left(-\delta I_{k}(t)-\mu I_{k}(t)\right) \\
\leq & \sum \frac{\varphi(k)}{\eta_{k}} P(k)\left[\lambda(k) \Theta(t) \eta_{k}+\frac{-(\beta+\mu)(\delta+\mu)}{\beta h} I_{k}(t)\right] \\
= & \Theta(t) \frac{1}{\beta h}((\beta+\mu)(\delta+\mu))\left(R_{0}-1\right) .
\end{aligned}
$$

When $R_{0}<1$, we can obtain $V(t) \leq 0$ for all $t \geq 0$, and that $V(t)=0$ only if $\Theta(t)=0$, that is, $I_{k}^{*}=0$. Combining with the second equation of system (4.1), it obviously follows that $E_{k}^{*}=0$ as $t \rightarrow+\infty$ for $k=1,2, \ldots, n$.

Due to $I_{k}^{*}=0$ and $E_{k}^{*}=0$, from the first equation for system (2.1), it follows that

$$
\frac{d S_{k}(t)}{d t}=b(k)+\delta m I_{k}(t)-\lambda(k) \Theta(t) S_{k}(t)-\gamma k \Theta(t) S_{k}(t)-\mu S_{k}(t) .
$$

By Lemma 4.1, we derive that

$$
\lim _{t \rightarrow \infty} \sup S_{k}(t) \leq \frac{b(k)}{\mu}=: S_{k}^{0} .
$$

For arbitrarily enough small $\varepsilon_{2}>0$, there exists $t_{2}>0$ such that $0 \leq E_{k}(t) \leq \varepsilon_{2}, 0 \leq I_{k}(t) \leq$ $\varepsilon_{2}$ for $t>t_{2}$. From the first equation (4.1), we have

$$
\frac{d S_{k}(t)}{d t} \geq b(k)+\delta m \varepsilon_{2}-\lambda(k) S_{k}(t) M \varepsilon_{2}-\gamma k S_{k}(t) M \varepsilon_{2}-\mu S_{k}(t),
$$

where $M=\frac{1}{\langle k\rangle} \sum_{i=1}^{n} k P(k)$, by Lemma 4.1, we have $\lim _{t \rightarrow+\infty} S_{k}(t) \geq \frac{b(k)+\delta m \varepsilon_{2}}{\mu+(\lambda(k)+\gamma k) M \varepsilon_{2}}$. Setting $\varepsilon_{2} \rightarrow 0$, it follows that $\lim _{t \rightarrow+\infty} S_{k}(t) \geq \frac{b(k)}{\mu}=S_{k}^{0}$. 
From (4.4) and (4.7), it is clear that $\lim _{t \rightarrow+\infty} S_{k}(t)=S_{k}^{0}=\frac{b(k)}{\mu}=\eta(k)$. This proves that the rumor-free equilibrium $E_{0}$ of system (4.1) is globally asymptotically stable when $R_{0}<1$. The proof is completed.

Next, the global attractivity of the rumor-prevailing equilibrium is discussed. The main result is given in the following theorem.

Theorem 4 Suppose that $\left(S_{k}(t), E_{k}(t), I_{k}(t)\right)$ is a solution of system (4.1) satisfying initial conditions $E_{k}(t)>0$ or $I_{k}(t)>0$. If $R_{0}>1$, then $\lim _{t \rightarrow \infty}\left(S_{k}(t), E_{k}(t), I_{k}(t)\right)=\left(S_{k}^{*}(t), E_{k}^{*}(t), I_{k}^{*}(t)\right)$, where $\left(S_{k}^{*}(t), E_{k}^{*}(t), I_{k}^{*}(t)\right)$ is the information-prevailing equilibrium of (4.1) satisfying (3.2) for $k=1,2, \ldots, n$.

Proof In the following, $k$ is fixed to be any integer in $(1,2, \ldots, n)$. By Theorem 4 , there exists a sufficiently small constant $\xi(0<\xi<1)$ and a larger enough constant $T>0$ such that $I_{i_{0}}(t) \geq \xi$ for $t>T$, therefore $\Theta(t)>\xi \Theta$ for $t>T$. Thus

$$
\Theta=\frac{1}{\langle k\rangle} \sum_{i=1} \frac{\varphi(i)}{\eta_{i}} P(i) I_{i}(t) \geq \frac{\varphi\left(i_{0}\right) P\left(i_{0}\right)}{\eta_{i_{0}}\langle k\rangle} \xi=\phi \xi>0,
$$

where $\phi=\frac{\varphi\left(i_{0}\right) P\left(i_{0}\right)}{\eta_{i_{0}}(k)}$. Submitting this into the equation of (4.1) gives

$$
S_{k}^{\prime}(t) \leq b(k)+\delta m\left(\eta_{k}-S_{k}(t)\right)-(\lambda(k)+\gamma k) \phi \xi S_{k}(t)-\mu S_{k}(t), \quad t>T .
$$

By Lemma 4.1, we derive that $\lim \sup _{t \rightarrow \infty} S_{k}(t) \leq \frac{b(k)+\delta m \eta_{k}}{(\lambda(k)+\gamma k) \phi \xi+\mu+\delta m}$, thus, for any given constant $0<\xi_{1}<\frac{(\lambda(k)+\gamma k) \phi \xi \eta_{k}}{2(\mu+\delta m+(\lambda(k)+\gamma k) \phi \xi)}$, there exists $t_{1}>T$ such that $S_{k}(t) \leq A_{k}^{(1)}-\xi_{1}$ for $t>t_{1}$, where

$$
A_{k}^{(1)}=\frac{b(k)+\delta m \eta_{k}}{(\lambda(k)+\gamma k) \phi \xi+\mu}+2 \xi_{1}<\eta_{k}
$$

Since $\Theta \leq \frac{1}{\langle k\rangle} \sum_{i=1} \varphi(i) P(i)=: \Phi$, we obtain from the second equation of system (4.1) that

$$
\begin{aligned}
E_{k}^{\prime}(t) & \leq \lambda(k)\left(\eta_{k}-E_{k}(t)-I_{k}(t)-R_{k}(t)\right)-(\beta+\mu) E_{k}(t) \\
& \leq \lambda(k) \Phi\left(\eta_{k}-E_{k}(t)\right)-(\beta+\mu) E_{k}(t) \\
& =\eta_{k} \lambda(k) \Phi-E_{k}(t)(\lambda(k) \Phi+\beta+\mu), \quad t>t_{1} .
\end{aligned}
$$

Hence, for any given constant $0<\xi_{2}<\min \left\{1 / 2, \xi_{1},(\mu+\beta) \eta_{k}[2(\lambda(k) \Phi+\mu+\beta)]^{-1}\right\}$, there exists $t_{2}>t_{1}$ such that $E_{k}(t) \leq B_{k}^{(1)}-\xi_{2}$ for $t>t_{2}$, where

$$
B_{k}^{(1)}=\frac{\lambda(k) \Phi \eta_{k}}{\lambda(k) \Phi+\mu+\beta}+2 \xi_{2}<\eta_{k}
$$

Then it follows from the third equation of (4.1) that

$$
I_{k}^{\prime}(t) \leq \beta h\left(\eta_{k}-I_{k}(t)\right)-(\mu+\delta) I_{k}(t)=\beta h \eta_{k}-(\mu+\delta+\beta h) I_{k}(t), \quad t>t_{2} .
$$


Similarly, for any given constant $0<\xi_{3}<\min \left\{1 / 3, \xi_{2},(\mu+\beta h) \eta_{k}[2(\mu+\delta+\beta h)]^{-1}\right\}$, there exists $t_{3}>t_{2}$ such that $I_{k}(t) \leq C_{k}^{(1)}-\xi_{3}$ for $t>t_{3}$, where

$$
C_{k}^{(1)}=\frac{\delta \eta_{k}}{\mu+\delta+\beta h}+2 \xi_{3}<\eta_{k}
$$

On the other hand, we substitute this into the first equation of (4.1)

$$
S_{k}^{\prime}(t) \geq b(k)+\delta m \eta_{k}-(\lambda(k)+\gamma k) \Phi S_{k}(t)-\mu S_{k}(t)-\delta m S_{k}(t), \quad t>T .
$$

So, for any given enough small constant $0<\xi_{4}<\min \left\{1 / 4, \xi_{3},\left(b(k)+\delta m \eta_{k}\right)[2((\lambda(k)+\right.$ $\left.\gamma k) \Phi+\mu+\delta m)]^{-1}\right\}$, there exists $t_{4}>t_{3}$ such that $S_{k}(t) \geq a_{k}^{(1)}+\xi_{4}$ for $t>t_{4}$, where

$$
a_{k}^{(1)}=\frac{b(k)+\delta m \eta_{k}}{(\lambda(k)+\gamma k) \Phi+\mu+\delta m}-2 \xi_{4}>0 .
$$

It follows that

$$
E_{k}^{\prime}(t) \geq \lambda(k) \phi \xi a_{k}^{(1)}-(\beta+\mu) E_{k}(t), \quad t>t_{4} .
$$

So, for any given enough small constant $0<\xi_{5}<\min \left\{1 / 5, \xi_{4},\left(\lambda(k) \phi \xi a_{k}^{(1)}\right)[2(\mu+\beta)]^{-1}\right\}$, there exists $t_{5}>t_{4}$ such that $E_{k}(t) \geq b_{k}^{(1)}+\xi_{5}$ for $t>t_{5}$, where

$$
b_{k}^{(1)}=\left(\lambda(k) \phi \xi a_{k}^{(1)}\right)(\mu+\beta)^{-1}-2 \xi_{5}>0 .
$$

The third equation of (2.1) implies that

$$
I_{k}^{\prime}(t) \geq \beta h b_{k}^{(1)}-(\mu+\delta) I_{k}(t), \quad t>t_{5} .
$$

So, for any given enough small constant $0<\xi_{6}<\min \left\{1 / 6, \xi_{5},\left(\beta h b_{k}^{(1)}\right)(2(\mu+\delta))^{-1}\right\}$, there exists $t_{6}>t_{5}$ such that $I_{k}(t) \geq c_{k}^{(1)}+\xi_{6}$ for $t>t_{6}$, where $c_{k}^{(1)}=\beta h b_{k}^{(1)}(\mu+\delta)^{-1}-2 \xi_{6}>0$.

Due to $\xi$ being a small positive constant, we can derive that $0<a_{k}^{(1)}<A_{k}^{(1)}<1,0<b_{k}^{(1)}<$ $B_{k}^{(1)}<1$, and $0<c_{k}^{(1)}<C_{k}^{(1)}<1$. Let

$$
q^{(j)}=\frac{1}{\langle k\rangle} \sum_{j=1}^{n} i P(i) c_{i}^{(j)}, \quad Q^{(j)} \frac{1}{\langle k\rangle} \sum_{j=1}^{n} i P(i) C_{i}^{(j)}, \quad j=1,2, \ldots
$$

We can easily get $0<q^{(j)} \leq \Theta(t) \leq Q^{(j)}<\Phi, t>t_{4}$.

Again, from the first equation of (4.1), we have

$$
S_{k}^{\prime}(t) \leq b(k)+\delta m \eta_{k}-(\lambda(k)+\gamma k) q^{(1)} S_{k}(t)-\mu S_{k}(t)-\delta m S_{k}(t), \quad t>t_{4} .
$$

Hence, for any given constant $0<\xi_{7}<\min \left\{1 / 7, \xi_{6}\right\}$, there exists $t_{7}>t_{6}$ such that

$$
S_{k}^{\prime}(t) \leq A_{k}^{(2)} \triangleq \min \left\{A_{k}^{(1)}-\xi_{1},\left(b(k)+\delta m \eta_{k}\right)\left[(\lambda(k)+\gamma k) q^{(1)}+\mu+\delta m\right]^{-1}+\xi_{7}\right\}, \quad t>t_{7} .
$$

Then, from the second equation of (4.1), we have

$$
E_{k}^{\prime}(t) \geq \lambda(k) Q^{(1)} A_{k}^{(2)}-(\mu+\beta) E_{k}(t), \quad t>t_{7} .
$$


So, for any given constant $0<\xi_{8}<\min \left\{1 / 8, \xi_{7}\right\}$, there exists $t_{8}>t_{7}$ such that

$$
E_{k}^{\prime}(t) \leq B_{k}^{(2)} \triangleq \min \left\{B_{k}^{(1)}-\xi_{2},\left(\lambda(k) Q^{(1)} A_{k}^{(2)}\right)(\mu+\beta)^{-1}+\xi_{8}\right\}, \quad t>t_{8} .
$$

Consequently, from the third equation of (4.1), we have

$$
I_{k}^{\prime}(t) \leq \beta h B_{k}^{(2)}-(\mu+\delta) I_{k}(t), \quad t>t_{8}
$$

Hence, for any given constant $0<\xi_{9}<\min \left\{1 / 9, \xi_{8}\right\}$, there exists $t_{9}>t_{8}$ such that

$$
I_{k}^{\prime}(t) \leq C_{k}^{(2)} \triangleq \min \left\{C_{k}^{(1)}-\xi_{3},\left(\beta h B_{k}^{(2)}\right)(\delta+\mu)^{-1}+\xi_{9}\right\}, \quad t_{9}>t_{8} .
$$

Turning back, one has

$$
S_{k}^{\prime}(t) \geq b(k)+\delta m \eta_{k}-(\lambda(k)+\gamma k) Q^{(2)} S_{k}(t)-\mu S_{k}(t)-\delta m S_{k}(t), \quad t>t_{9} .
$$

So, for any given enough small constant $0<\xi_{10}<\min \left\{1 / 10, \xi_{9},\left(b(k)+\delta m \eta_{k}\right)[2(\lambda(k)+\right.$ $\left.\left.\gamma k) Q^{(2)}+\mu+\delta m\right]^{-1}\right\}$, there exists $t_{10}>t_{9}$ such that $S_{k}(t) \geq a_{k}^{(2)}+\xi_{10}$ for $t>t_{10}$, where

$$
\left.a_{k}^{(2)}=\max \left\{a_{k}^{(1)}+\xi_{4},\left(b(k)+\delta m \eta_{k}\right)(\lambda(k)+\gamma k) Q^{(2)}+\mu+\delta m\right)^{-1}-2 \xi_{10}\right\} .
$$

It follows that

$$
E_{k}^{\prime}(t) \geq \lambda(k) q^{(1)} a_{k}^{(2)}-(\beta+\mu) E_{k}(t), \quad t>t_{10} .
$$

So, for any given enough small constant $0<\xi_{11}<\min \left\{1 / 11, \xi_{10}, \lambda(k) q^{(1)} a_{k}^{(2)}+[2(\mu+\right.$ $\left.\beta)]^{-1}\right\}$, there exists $t_{11}>t_{10}$ such that $E_{k}(t) \geq b_{k}^{(2)}+\xi_{11}$ for $t>t_{10}$, where

$$
b_{k}^{(2)}=\max \left\{b_{k}^{(1)}+\xi_{5}, \lambda(k) q^{(1)} a_{k}^{(2)}+[2(\mu+\beta)]^{-1}-2 \xi_{11}\right\} .
$$

The third equation of (4.1) implies that

$$
I_{k}^{\prime}(t) \geq \beta h b_{k}^{(2)}-(\mu+\delta) I_{k}(t), \quad t>t_{11}
$$

So, for any given enough small constant $0<\xi_{12}<\min \left\{1 / 12, \xi_{11},\left[\beta h b_{k}^{(2)}\right](2(\mu+\delta))^{-1}\right\}$, there exists $t_{12}>t_{11}$ such that $I_{k}(t) \geq c_{k}^{(2)}+\xi_{12}$ for $t>t_{12}$, where

$$
c_{k}^{(2)}=\max \left\{\left[c_{k}^{(1)}+\xi_{6}, \beta h b_{k}^{(2)}\right](\mu+\delta)^{-1}-2 \xi_{12}\right\} .
$$

Repeating the above analyses and calculations, we get six sequences $A_{k}^{(i)}, B_{k}^{(i)}, C_{k}^{(i)}, a_{k}^{(i)}, b_{k}^{(i)}$, $c_{k}^{(i)}, i=1,2, \ldots$. Due to the first three being monotone decreasing sequences and the last three being monotone increasing ones, there exists a sufficiently large positive integer $L \geq$ 
2 such that $l \geq L$ :

$$
\begin{aligned}
& A_{k}^{(l)}=\frac{b(k)+\delta m \eta_{k}}{(\lambda(k)+\gamma k) q^{(l-1)}+\mu+\delta m}+\xi_{6 l-5}, \quad B_{k}^{(l)}=\frac{\lambda(k) Q^{(l-1)} A_{k}^{(l)}}{\mu+\beta}+\xi_{6 l-4}, \\
& C_{k}^{(l)}=\frac{\beta h B_{k}^{(l)}}{\delta+\mu}+\xi_{6 l-3}, \quad a_{k}^{(l)}=\frac{b(k)+\delta m \eta_{k}}{(\lambda(k)+\gamma k) Q^{(l)}+\mu+\delta m}+\xi_{6 l-2}, \\
& b_{k}^{(l)}=\frac{\lambda(k) q^{(l-1)} a_{k}^{(l)}}{\mu+\beta}+\xi_{6 l-1}, \quad c_{k}^{(l)}=\frac{\beta h b_{k}^{(l)}}{\delta+\mu}+\xi_{6 l l} .
\end{aligned}
$$

We can easily get that

$$
a_{k}^{(l)} \leq S_{k}(t) \leq A_{k}^{(l)}, \quad b_{k}^{(l)} \leq E_{k}(t) \leq B_{k}^{(l)}, \quad c_{k}^{(l)} \leq I_{k}(t) \leq C_{k}^{(l)}, \quad t>t_{6 l} .
$$

Since the sequential limits of (4.21) exist, $\operatorname{let}_{\lim } \rightarrow \infty \Delta_{k}^{(l)}=\Delta_{k}$, where $\Delta_{k}^{(l)} \in\left\{A_{k}^{(l)}, B_{k}^{(l)}, C_{k}^{(l)}\right.$, $\left.a_{k}^{(l)}, b_{k}^{(l)}, c_{k}^{(l)}, Q_{k}^{(l)}, q_{k}^{(l)}\right\}$ and $\Delta_{k} \in\left\{A_{k}, B_{k}, C_{k}, a_{k}, b_{k}, c_{k}, Q_{k}, q_{k}\right\}$.

Noting that $0<\xi_{1}<1 / l$, one has $\xi_{1} \rightarrow 0$ as $l \rightarrow \infty$. In the six sequences of (4.21), by taking $l \rightarrow \infty$, it follows from (4.21) that

$$
\begin{array}{ll}
A_{k}=\frac{b(k)+\delta m \eta_{k}}{(\lambda(k)+\gamma k) q+\mu+\delta m}, & B_{k}=\frac{\lambda(k) Q A_{k}}{\mu+\beta},
\end{array}
$$

where

$$
q=\frac{1}{\langle k\rangle} \sum_{i=1}^{n} i P(i) c_{i}, \quad Q=\frac{1}{\langle k\rangle} \sum_{i=1}^{n} i P(i) C_{i} .
$$

Further,

$$
\begin{aligned}
C_{k}^{(l)} & =\frac{\beta h \lambda(k) Q\left(b(k)+\delta m \eta_{k}\right)}{(\delta+\mu)(\mu+\beta)((\lambda(k)+\gamma k) q+\mu+\delta m)}, \\
c_{k}^{(l)} & =\frac{\beta h \lambda(k) q\left(b(k)+\delta m \eta_{k}\right)}{(\delta+\mu)(\mu+\beta)((\lambda(k)+\gamma k) Q+\mu+\delta m)} .
\end{aligned}
$$

Substituting (4.24) into $q$ and $Q$, respectively, one has

$$
\begin{aligned}
& 1=\frac{\beta h(\mu+\delta m)}{\langle k\rangle(\delta+\mu)(\mu+\beta)} \sum_{i=1}^{n} \frac{\varphi(k) P(k) \lambda(k)}{((\lambda(k)+\gamma k) Q+\mu+\delta m)}, \\
& 1=\frac{\beta h(\mu+\delta m)}{\langle k\rangle(\delta+\mu)(\mu+\beta)} \sum_{i=1}^{n} \frac{\varphi(k) P(k) \lambda(k)}{((\lambda(k)+\gamma k) q+\mu+\delta m)} .
\end{aligned}
$$

By subtracting the above two equations, we arrive at

$$
0=\frac{\beta h(\mu+\delta m)(Q-q)}{\langle k\rangle(\delta+\mu)(\mu+\beta)} \sum_{i=1}^{n} \frac{\varphi(k) P(k) \lambda(k)(\lambda(k)+\gamma k)}{((\lambda(k)+\gamma k) Q+\mu+\delta m)((\lambda(k)+\gamma k) q+\mu+\delta m)} .
$$


It is obvious that $q=Q$, so $\frac{1}{\langle k\rangle} \sum_{i=1}^{n} \frac{\varphi(k)}{\eta_{k}} P(k)\left(C_{k}-c_{k}\right)=0$, which sees that $C_{k}=c_{k}$ for $i=1,2, \ldots, n$. From (4.24) and (4.25), it follows that

$$
\lim _{t \rightarrow \infty} S_{k}(t)=A_{k}=a_{k}, \quad \lim _{t \rightarrow \infty} E_{k}(t)=B_{k}=b_{k}, \quad \lim _{t \rightarrow \infty} I_{k}(t)=C_{k}=c_{k} .
$$

Finally, substituting $q=Q$ into (4.22), in view of (3.2) and (4.23), we obtain $S_{k}=S_{k}^{*}, E_{k}=$ $E_{k}^{*}$, and $I_{k}=I_{k}^{*}$. The proof is completed.

\section{The modified SEIR model with feedback mechanism}

Rumor has a sudden and fast spreading speed, it has bad influence on the normal social stability. Due to the fact that the internet rumors are difficult to identify and bewitch, it is easy to cause serious social problems and even cause social unrest and political instability. However, due to the rapid development of network technology, the information spreading on the network will be further verified, which will weaken the spread of rumors, and this can be described as a feedback mechanism. Based on the above observations and model (2.1), we present the modified model with feedback mechanism in social networks:

$$
\left\{\begin{aligned}
\frac{d S_{k}(t)}{d t}= & b(k)+\delta m I_{k}(t)-\lambda(k) \Theta(t)(1-\alpha \Theta(t)) S_{k}(t) \\
& -\gamma k \Theta(t)(1-\alpha \Theta(t)) S_{k}(t)-\mu S_{k}(t) \\
\frac{d E_{k}(t)}{d t}= & \lambda(k) \Theta(t)(1-\alpha \Theta(t)) S_{k}(t)-\mu E_{k}(t)-\beta E_{k}(t) \\
\frac{d I_{k}(t)}{d t}= & \beta h E_{k}(t)-\delta I_{k}(t)-\mu I_{k}(t) \\
\frac{d R_{k}(t)}{d t}= & \gamma k \Theta(t)(1-\alpha \Theta(t)) S_{k}(t)+\delta(1-m) I_{k}(t)+\beta(1-h) E_{k}(t)-\mu R_{k}(t)
\end{aligned}\right.
$$

where $\alpha$ is the positive parameter called 'fear factor', which is determined by the fear degree of people to the rumor spreading. $\Theta(t)$ denotes the probability of a susceptible contacting an infected at time $t$, which satisfies the relation

$$
\Theta(t)=\frac{1}{\langle k\rangle} \sum_{i=1}^{n} \frac{\varphi(k)}{\eta_{k}} P(k) I_{k}(t) .
$$

Here, $P(i \mid k)=i P(k) /\langle k\rangle, \lambda(k)(1-\alpha \Theta(t)) S_{k}(t) \Theta(t)$ represents the proportion of individuals who having acquired infection became exposed individuals. The spreading speed will decrease when $\alpha$ becomes lower, which is consistent with the actual prevalence law of rumor spreading.

Theorem 5 Consider model (5.1), define $R_{1}=\frac{\langle\varphi(k) \lambda(k)\rangle \beta h}{\langle k\rangle(\delta+\mu)(\beta+\mu)}$, then the following statements hold:

(1) If $R_{1}<1$, there always exists a rumor-free equilibrium $E_{1}\left(\eta_{k}, 0,0,0\right)$.

(2) There is a rumor-prevailing equilibrium $E_{1+}\left(S_{k}^{*}, E_{k}^{*}, I_{k}^{*}, R_{k}^{*}\right)$ if $R_{1}>1$. 

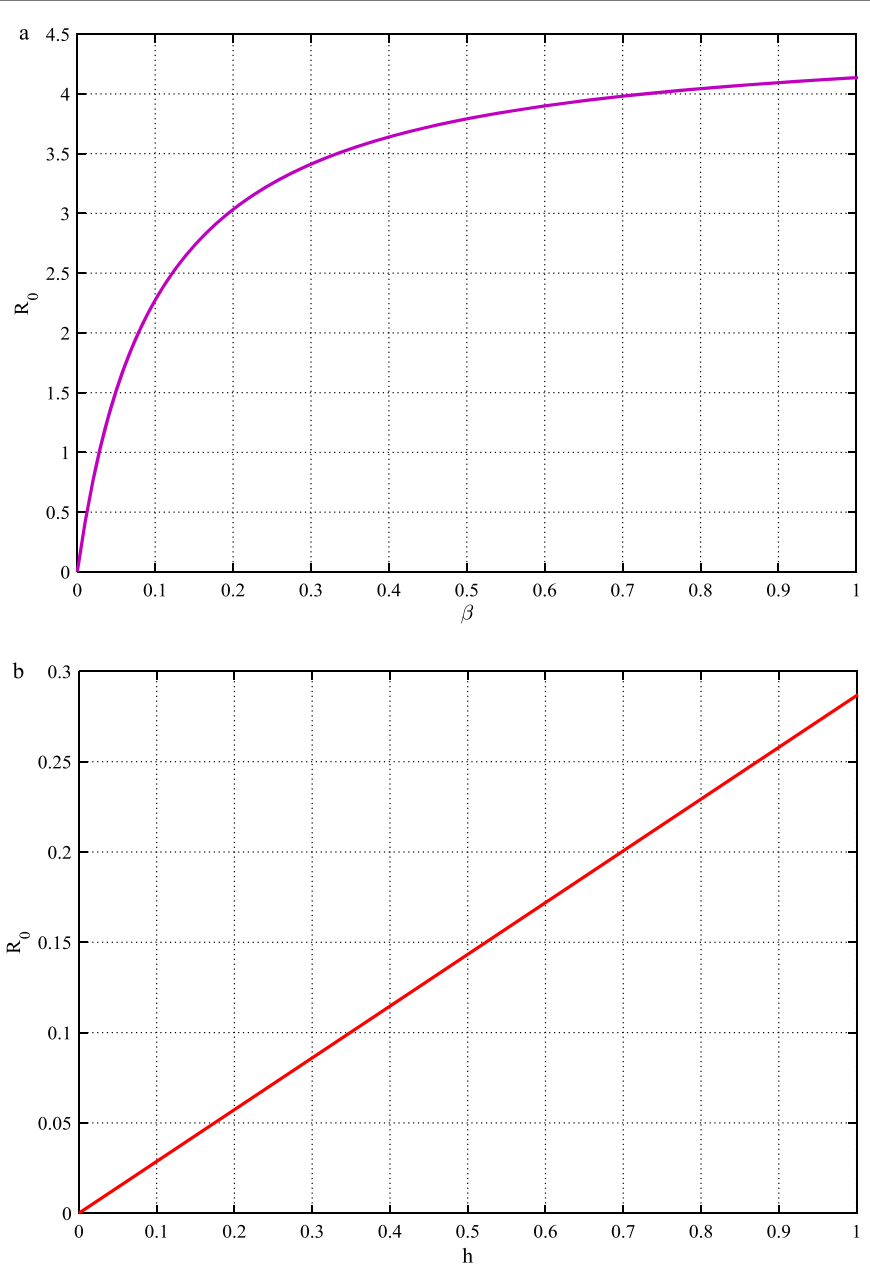

Figure 2 The relationship between the basic reproduction number $R_{0}$ and the parameters on social networks

Proof To get the rumor-prevailing equilibrium solution $E_{1+}\left(S_{k}^{*}, E_{k}^{*}, I_{k}^{*}, R_{k}^{*}\right)$, we need to make the right-hand side of system (5.1) equal to zero, it should satisfy

$$
\left\{\begin{array}{l}
b(k)+\delta m I_{k}^{*}-\lambda(k) \Theta^{*}\left(1-\alpha \Theta^{*}\right) S_{k}^{*}-\gamma k \Theta^{*}\left(1-\alpha \Theta^{*}\right) S_{k}^{*}-\mu S_{k}^{*}=0, \\
\lambda(k) \Theta^{*}\left(1-\alpha \Theta^{*}\right) S_{k}^{*}-\mu E_{k}^{*}-\beta E_{k}^{*}=0, \\
\beta h E_{k}^{*}-\delta I_{k}^{*}-\mu I_{k}^{*}=0, \\
\gamma k \Theta^{*}\left(1-\alpha \Theta^{*}\right) S_{k}^{*}+\delta(1-m) I_{k}^{*}+\beta(1-h) E_{k}^{*}-\mu R_{k}^{*}=0, \\
S_{k}^{*}+E_{k}^{*}+I_{k}^{*}+R_{k}^{*}=\eta_{k},
\end{array}\right.
$$

where $\Theta^{*}=\frac{1}{\langle k\rangle} \sum_{i=1}^{n} \frac{\varphi(k)}{\eta_{k}} p(k) I_{k}^{*}(t)$. One has

$$
\left\{\begin{array}{l}
S_{k}^{*}=\frac{(\mu+\delta)(\beta+\mu)}{\lambda(k) \beta h \Theta^{*}\left(1-\alpha \Theta^{*}\right)} I_{k}^{*}, \\
E_{k}^{*}=\frac{\mu+\delta}{\beta h} I_{k}^{*}, \\
R_{k}^{*}=\frac{\gamma k(\beta+\mu)(\mu+\delta)+\lambda(k) \beta((\mu+\delta)-h(\mu+\delta m))}{\lambda(k) \beta h \mu} I_{k}^{*} .
\end{array}\right.
$$



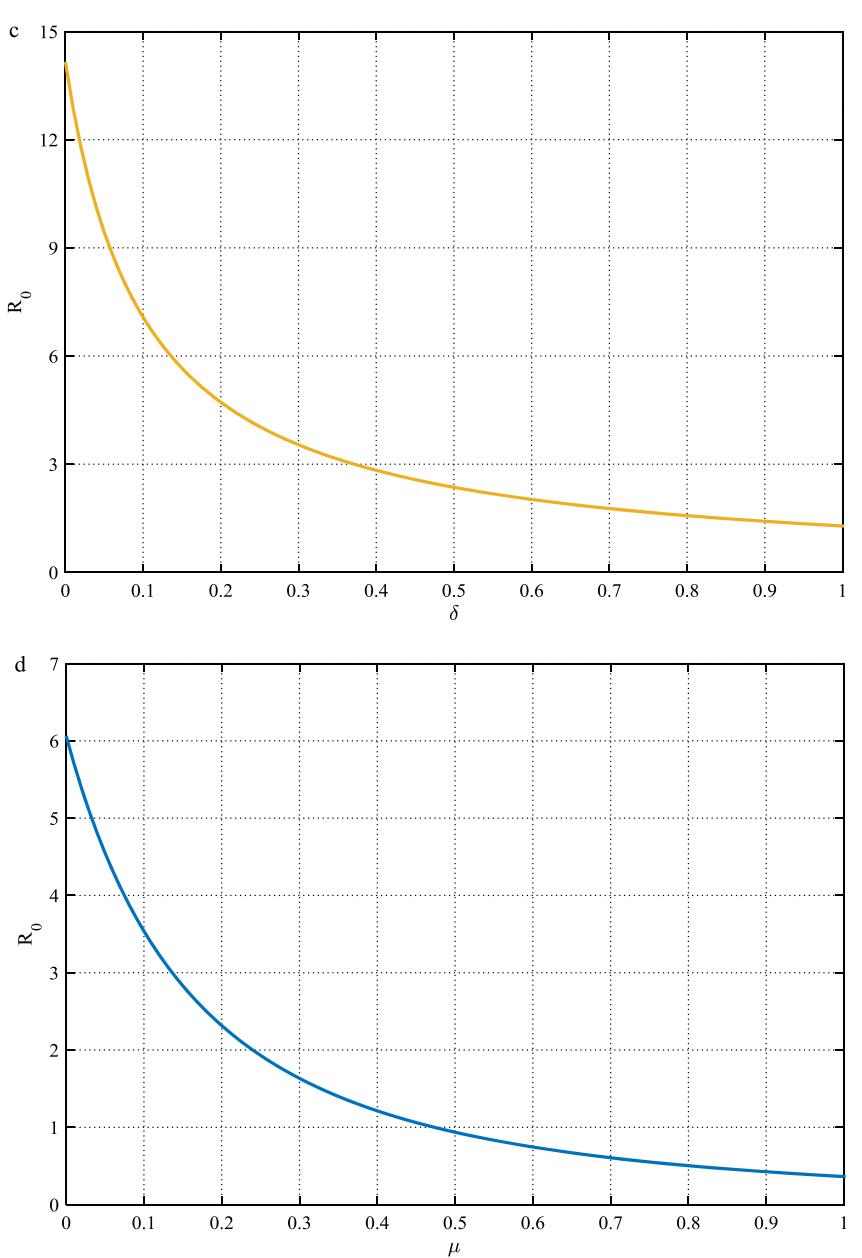

Figure 2 Continued

Considering the following normalization condition $S_{k}(t)+E_{k}(t)+I_{k}(t)+R_{k}(t)=\eta_{k}$ for all $k$, we obtain

$$
I_{k}^{*}=\frac{\mu \lambda(k) \Theta^{*}\left(1-\alpha \Theta^{*}\right) \beta h \eta_{k}}{\left(\mu+\gamma k \Theta^{*}\left(1-\alpha \Theta^{*}\right)\right)(\beta+\mu)(\delta+\mu)+\lambda(k) \Theta^{*}\left(1-\alpha \Theta^{*}\right)((\beta+\mu)(\delta+\mu)-\beta h \delta m)} .
$$

Inserting (5.5) into (5.2), we have the self-consistency equation

$$
\begin{aligned}
\Theta^{*}= & \frac{1}{\langle k\rangle} \sum_{i=1} \varphi(k) P(k) \\
& \times \frac{\Theta^{*}\left(1-\alpha \Theta^{*}\right) \lambda(k) \beta h}{\left(\gamma k \Theta^{*}\left(1-\alpha \Theta^{*}\right)+\mu\right)(\beta+\mu)(\delta+\mu)+\lambda(k) \Theta^{*}\left(1-\alpha \Theta^{*}\right)((\beta+\mu)(\delta+\mu)-\beta h \delta m)} \\
= & : f\left(\Theta^{*}\right) .
\end{aligned}
$$

Clearly, $\Theta^{*}=0$ is the solution of (5.6). To ensure that (5.6) has a nontrivial solution, i.e., $0<\Theta^{*} \leq 1$, the following conditions must be satisfied:

$$
\left.\frac{d f\left(\Theta^{*}\right)}{d \Theta^{*}}\right|_{\Theta^{*}=0}>1 \text { and } f(1) \leq 1 \text {, }
$$


which indicates that $\frac{\langle\varphi(k) \lambda(k)\rangle}{\langle k\rangle} \frac{\beta h}{(\delta+\mu)(\beta+\mu)}>1$, so we can get the basic reproductive number

$$
R_{1}=\frac{\beta h}{(\delta+\mu)(\beta+\mu)} \frac{\langle\varphi(k) \lambda(k)\rangle}{\langle k\rangle} .
$$

So, a nontrivial solution exists if and only if $R_{1}>1$.

Remark The feedback mechanism parameter $\alpha$ cannot change the basic reproductive number, but it can reduce the prevailing level and weaken the rumor spreading.

Theorem 6 When $R_{1}<1$, the rumor-free equilibrium is globally asymptotically stable; when $R_{1}>1$, system (5.1) is permanent, there exists $\varepsilon>0$ such that

$$
\lim \inf _{x \rightarrow \infty}\left\{S_{k}(t), E_{k}(t), I_{k}(t)\right\}_{k=1}^{n} \geq \varepsilon
$$

where $\left(S_{k}(t), E_{k}(t), I_{k}(t)\right)$ is any solution of $(5.3)$ satisfying $(5.1)$ and $E_{k}(0)>0$ or $I_{k}(0)>0$.

Proof Let $S_{k}(t)=\eta_{k}+x_{k}(t), E_{k}(t)=y_{k}(t), I_{k}(t)=z_{k}(t), k=1,2, \ldots, n$, where $\left(x_{k}(t), y_{k}(t), z_{k}(t)\right)$ is a small perturbation of $E_{1}$. Now we consider the linearized system at $E_{1}$ :

$$
\left\{\begin{array}{l}
\frac{d x_{k}(t)}{d t}=-\mu x_{k}(t)+\delta m z_{k}(t)-(\lambda(k)+\gamma k) \frac{1}{\langle k\rangle} \sum \varphi(k) P(k) z_{i}(t), \\
\frac{d y_{k}(t)}{d t}=\gamma k \frac{1}{\langle k\rangle} \sum \varphi(k) P(k) z_{i}(t)-\mu y_{k}(t)-\beta y_{k}(t), \\
\frac{d z_{k}(t)}{d t}=\beta h y_{k}(t)-\delta z_{k}(t)-\mu z_{k}(t)
\end{array}\right.
$$

which can be written as

$$
\frac{d}{d t}=\left(\begin{array}{c}
x_{1} \\
y_{1} \\
z_{1} \\
x_{2} \\
y_{2} \\
z_{2} \\
\vdots \\
x_{n} \\
y_{n} \\
z_{n}
\end{array}\right)=Q\left(\begin{array}{c}
x_{1} \\
y_{1} \\
z_{1} \\
x_{2} \\
y_{2} \\
z_{2} \\
\vdots \\
x_{n} \\
y_{n} \\
z_{n}
\end{array}\right),
$$

where matrix $Q$ is

$$
\left(\begin{array}{cccccccccc}
-\mu & 0 & \delta m-A_{1} l_{1} & 0 & 0 & -A_{1} l_{2} & \cdots & 0 & 0 & -A_{1} l_{n} \\
0 & -\mu-\beta & B_{1} l_{1} & 0 & 0 & B_{1} l_{2} & \cdots & 0 & 0 & B_{1} l_{n} \\
0 & \beta h & -\delta-\mu & 0 & 0 & 0 & \cdots & 0 & 0 & 0 \\
0 & 0 & -A_{2} l_{1} & -\mu & 0 & \delta m-A_{2} l_{2} & \cdots & 0 & 0 & -A_{2} l_{n} \\
0 & 0 & B_{2} l_{1} & 0 & -\mu-\beta & B_{2} l_{2} & \cdots & 0 & 0 & B_{2} l_{n} \\
0 & 0 & 0 & 0 & \beta h & -\delta-\mu & \cdots & 0 & 0 & 0 \\
\vdots & \vdots & \vdots & \vdots & \vdots & \vdots & \ddots & \vdots & \vdots & \vdots \\
0 & 0 & -A_{n} l_{1} & 0 & 0 & -A_{n} l_{2} & \cdots & -\mu & 0 & \delta m-A_{n} l_{n} \\
0 & 0 & B_{n} l_{1} & 0 & 0 & B_{n} l_{2} & \cdots & 0 & -\mu-\beta & B_{n} l_{n} \\
0 & 0 & 0 & 0 & 0 & 0 & \cdots & 0 & \beta h & -\delta-\mu
\end{array}\right)
$$



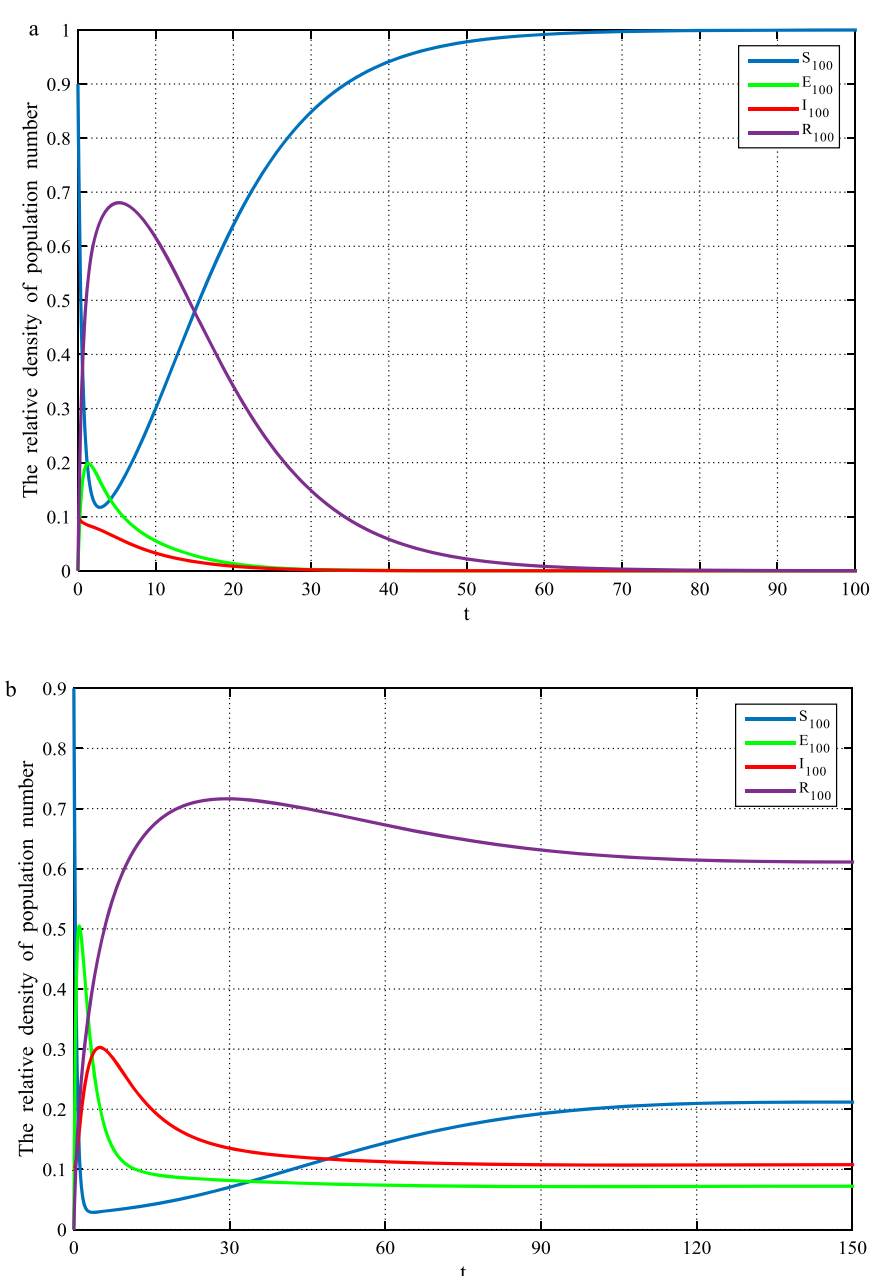

Figure 3 Each compartment population changes over time when $R_{0}<1(\mathbf{a})$ and $R_{0}>1$ (b)

To simplify the matrix, we rewrite it as follows:

$$
Q=\left(\begin{array}{ccc}
N_{11} & \cdots & N_{1 n} \\
\vdots & \ddots & \vdots \\
N_{n 1} & \cdots & N_{n n}
\end{array}\right),
$$

where

$$
\begin{aligned}
N_{11} & =\left(\begin{array}{ccc}
-\mu & 0 & \delta m-A_{1} l_{1} \\
0 & -\mu-\beta & B_{1} l_{1} \\
0 & \beta h & -\delta-\mu
\end{array}\right), \\
N_{1 n} & =\left(\begin{array}{ccc}
0 & 0 & A_{1} l_{n} \\
0 & 0 & B_{1} l_{n} \\
0 & 0 & 0
\end{array}\right),
\end{aligned}
$$



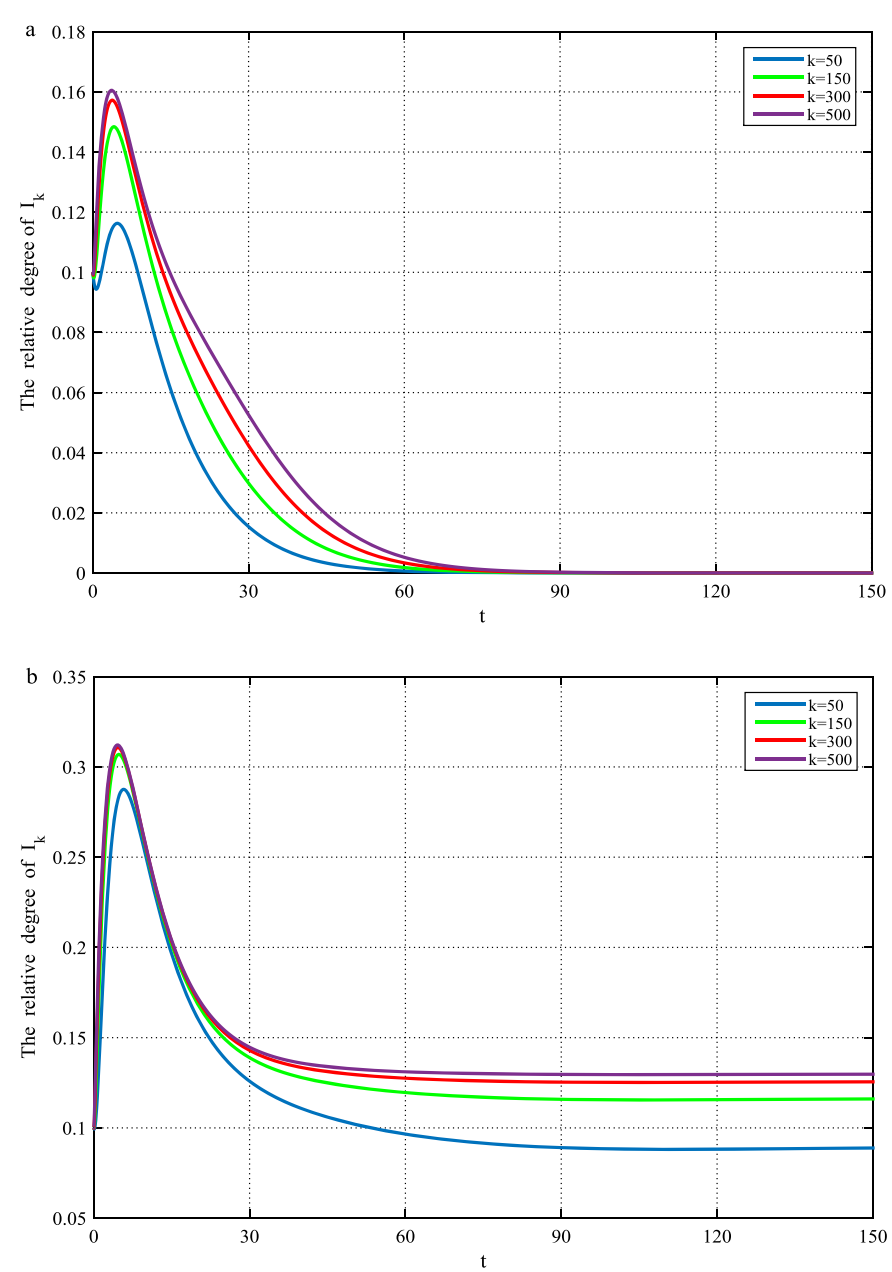

Figure 4 Dynamic behavior of $I_{k}$ in rumor-free equilibrium (a) and rumor-prevailing equilibrium (b) with different degree

$$
\begin{aligned}
N_{n 1} & =\left(\begin{array}{ccc}
0 & 0 & A_{n} l_{1} \\
0 & 0 & B_{n} l_{1} \\
0 & 0 & 0
\end{array}\right), \\
N_{n n} & =\left(\begin{array}{ccc}
-\mu & 0 & \delta m-A_{n} l_{n} \\
0 & -\mu-\beta & B_{n} l_{n} \\
0 & \beta h & -\delta-\mu
\end{array}\right),
\end{aligned}
$$

in which $A_{k}=\frac{\lambda(k)+\gamma k}{\langle k\rangle}, B_{k}=\frac{\lambda(k)}{\langle k\rangle}, l_{k}=\varphi(k) P(k)$. A direct calculation leads to the characteristic polynomial of the rumor-free equilibrium in the following form:

$$
(x+\mu)^{n}(x+(\mu+\beta))^{n-1}(x+(\mu+\delta))^{n-1}\left(x^{2}+p x+q\right)=0,
$$

where $p=2 \mu+\delta+\beta, q=(\beta+\mu)(\delta+\mu)-\beta h \sum_{i=1}^{n} \varphi(i) P(i)$.

It is obvious that $p>0$ and $R_{1}<1$ is equivalent to $q>0$. Therefore, there exists a unique positive eigenvalue $x$ of $Q$ if and only if $R_{1}>1$; otherwise, if $R_{1}<1$, all real-valued eigenvalues of $Q$ are negative. By the Perron-Frobenius theorem, this implies that the maximal real 

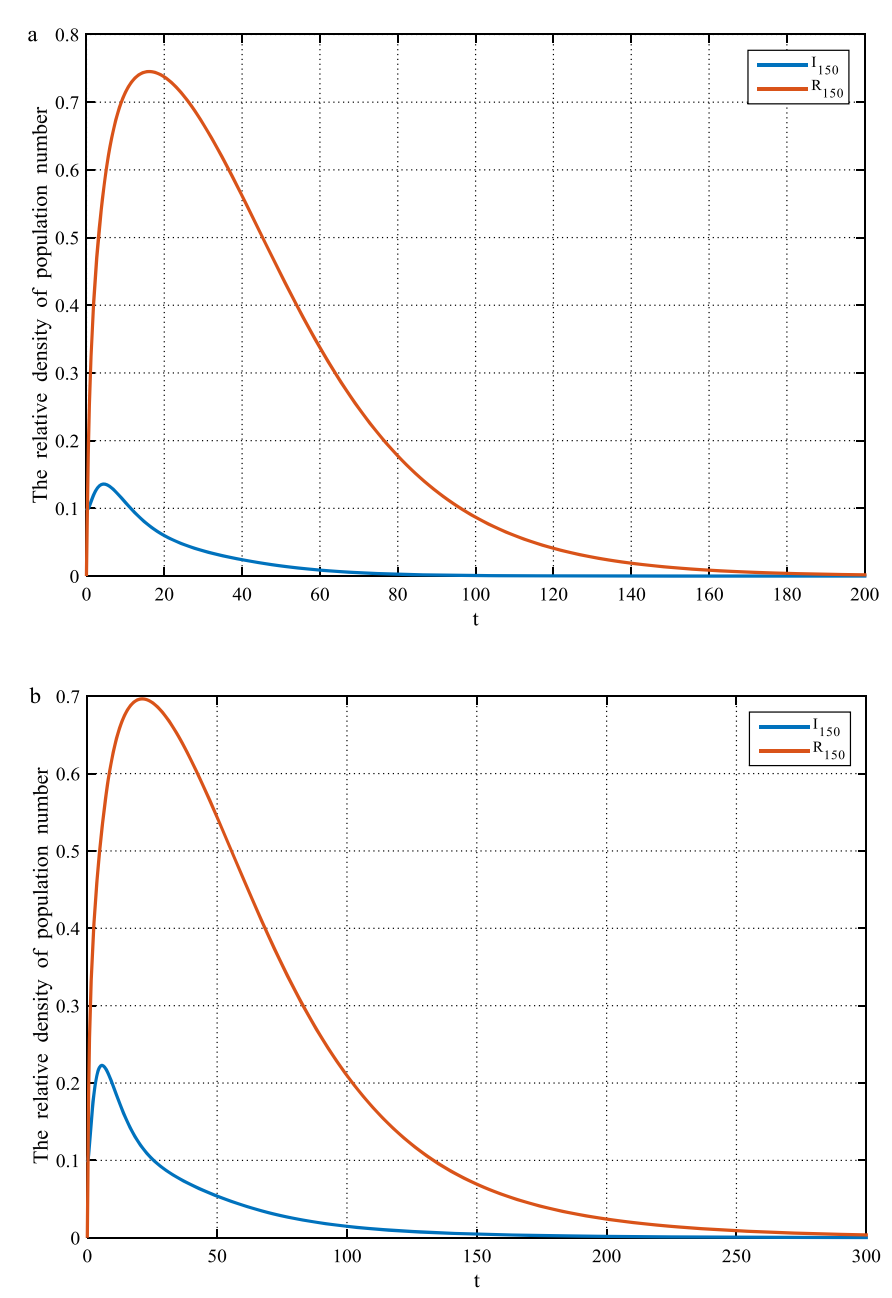

Figure 5 Density of $/_{150}$ and $R_{150}$ versus varying over different attractive parameter $h$

part of all eigenvalues of $Q$ is positive if and only if $R_{1}>1$. Then a theorem of Lajmanovich and York [45] yields the results of this theorem. The proof is completed.

Conjecture Suppose that $\left(S_{k}(t), E_{k}(t), I_{k}(t)\right)$ is a solution of $(5.3)$ with $S_{k}(0)>0, E_{k}(0)>0$ and $I_{k}(0)>0$. If $R_{1}>1$, then $\liminf _{x \rightarrow \infty}\left\{S_{k}(t), E_{k}(t), I_{k}(t)\right\}=\left(S_{k}^{*}, E_{k}^{*}, I_{k}^{*}\right)$, where $\left(S_{k}^{*}, E_{k}^{*}, I_{k}^{*}\right)$ is the unique alcoholism equilibrium of (5.3).

Remark We have great difficulty in proving the global stability of $E_{1+}$. Then we only carry out simulations to test our conjecture (Fig. 9). It is still an open problem to prove the global stability of $E_{1+}$.

\section{Simulation results and analysis}

First, we perform some sensitivity analysis of the basic reproduction number $R_{0}$ in terms of the model parameters on social networks. Obviously,

$$
\frac{\partial R_{0}}{\partial h}=\frac{\langle\lambda(k) \varphi(k)\rangle}{\langle k\rangle} \frac{\beta}{[(\delta+\mu)(\beta+\mu)]^{2}}, \quad \frac{\partial R_{0}}{\partial \delta}=\frac{\langle\lambda(k) \varphi(k)\rangle}{\langle k\rangle} \frac{-\beta h(\beta+\mu)}{[(\delta+\mu)(\beta+\mu)]^{2}},
$$



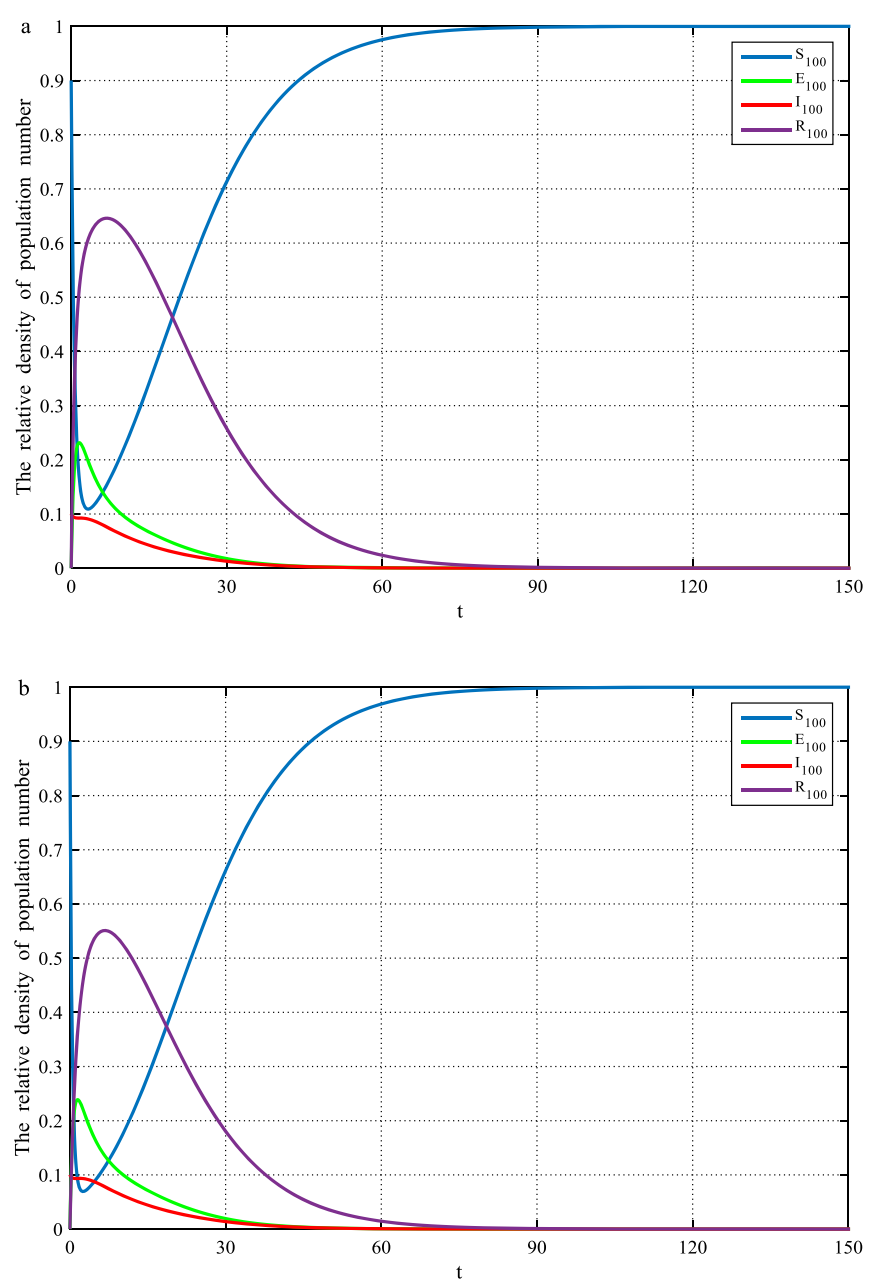

Figure 6 Each compartment population changes over time with different forget rate $\delta$

$$
\frac{\partial R_{0}}{\partial \beta}=\frac{\langle\lambda(k) \varphi(k)\rangle}{\langle k\rangle} \frac{\mu h(\delta+\mu)}{[(\delta+\mu)(\beta+\mu)]^{2}}, \quad \frac{\partial R_{0}}{\partial \mu}=\frac{\langle\lambda(k) \varphi(k)\rangle}{\langle k\rangle} \frac{-(\delta+2 \mu+\mu)}{[(\delta+\mu)(\beta+\mu)]^{2}} .
$$

We can find some interesting results, which have been showed in Fig. 2. In Fig. 2(a) and (b), it can be seen that big $\beta$ or $h$ can lead to large $R_{0}$. That is to say, the larger infected or the higher attractiveness of rumor can increase the chance of rumor spreading. From Fig. 2(c) and (d), $R_{0}$ increases as $\delta$ or $\mu$ decreases. At the same time, variance of degree distribution $\langle\varphi(k) \lambda(k)\rangle$ manifests the diversity in contact patterns. Particularly, the ratio $\langle\varphi(k) \lambda(k)\rangle /\langle k\rangle$ is the parameter defining the level of heterogeneity of the network [26].

Next, we carry out Runge-Kutta method simulations to investigate the dynamics of model (2.1) on both artificial and real networks. We take the degree distribution to be $P(k)=c k^{-l}(2<l \leq 3)$, in which $l=3$ and $c$ satisfies $\sum_{k=1}^{n} P(k)=1, n=1000$. We choose $\lambda(k)=\lambda k, \varphi(k)=k, b(k)=b / n$.

In Fig. 3(a), the parameters are chosen as $b=0.1, \lambda=0.15, \gamma=0.1, \delta=0.2, \beta=0.25, m=$ $0.1, h=0.4$, thus the basic reproduction number $R_{0}=0.65$. It is shown that when $R_{0}<$ 1 , the rumor spreading will disappear, even for a large fraction of the infected nodes at the beginning. And we can also see $I_{k} \rightarrow 0$ as $t \rightarrow \infty$. It suggests that the rumor-free 

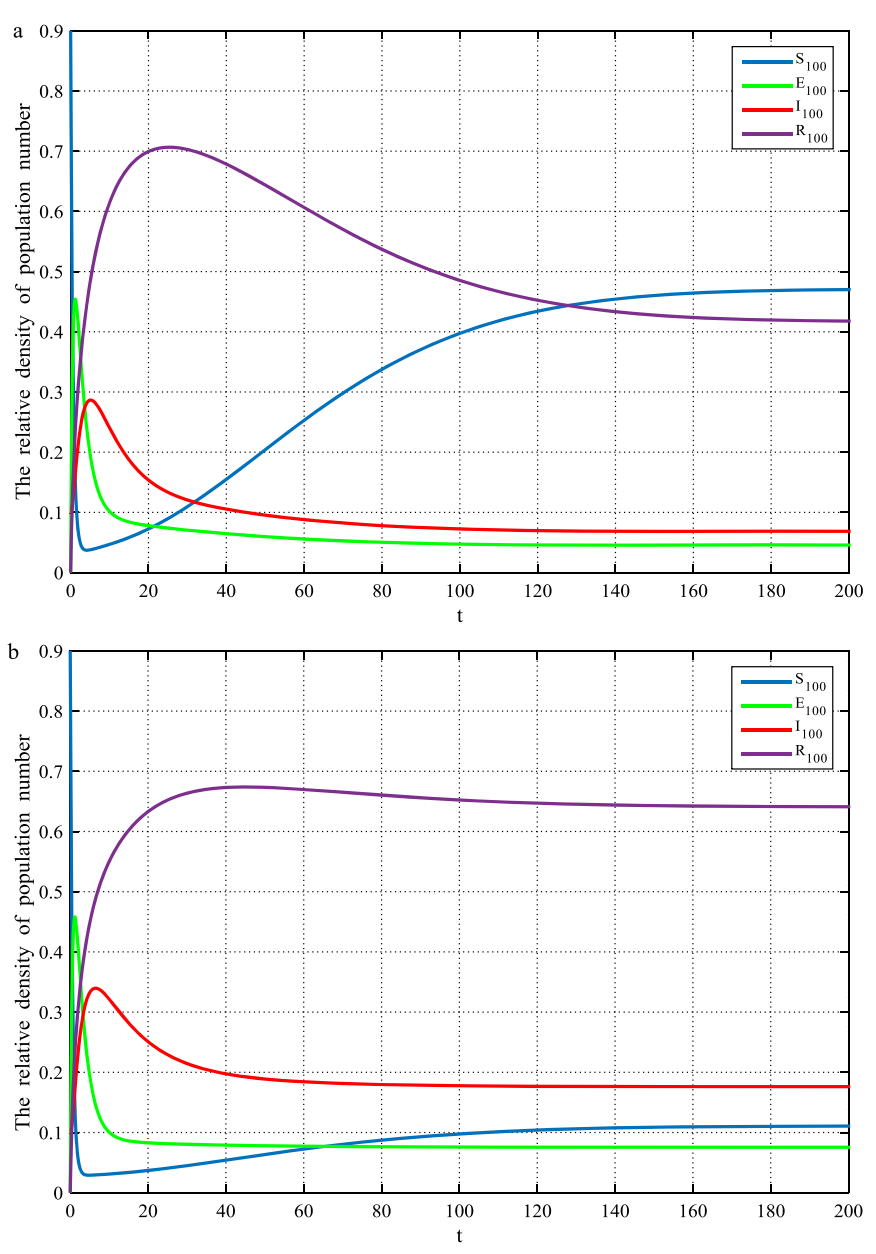

Figure 7 Each compartment population changes over time with different fuzzy parameter $m$

equilibrium is globally asymptotically stable when $R_{0}<1$, in agreement with Theorem 2 . In Fig. 3(b), the parameters are chosen as $b=0.04, \lambda=0.35, \gamma=0.1, \delta=0.2, \beta=0.3, m=$ $0.2, h=0.6$, the basic reproduction number is $R_{0}=3.51$. It is shown that when $R_{0}>1$, even for a small fraction of the infected nodes at the beginning, the rumor is permanent on the network, in accord with Theorem 2.

Figure 4 shows the dynamical behavior of $I_{100}$ in $R_{0}<1$ (a) and $R_{0}>1$ (b) with different degree. We find that the larger degree leads to a larger value of the spreading level.

Figure 5 shows the effect of attractive parameter $h$ to $I_{150}$ and $R_{150}$. We choose the parameters $h=0.1$ in Fig. 5(a) and $h=0.4$ in Fig. 5(b). We can find that the greater attraction of the rumor, the greater people who spread rumor and the fewer people who recover rumor.

Figures 6 and 7 show the effect of forget rate $\delta$ and fuzzy parameter $m$ to each population. One can see that both forget rate $\delta$ and fuzzy parameter $m$ have great influence on $S_{k}$ and $R_{k}$. In Fig. 6, we choose $\delta=0.1$ in (a) and $\delta=0.05$ in (a), it shows that the forget rate $\delta$ has a positive effect on $S_{k}$ and $R_{k}$. In Fig. 7, we choose $m=0.15$ in (a) and $m=0.4$ in (a), we find that increasing fuzzy parameter $m$ can decrease the level of recovered. In the real world, the fuzzier the rumor is, the more curious people will be. This fact causes a secondary rumor diffusion. Thus, if we want to reduce the final rumor size, the authoritative organizations or media should give precise and clear information. 

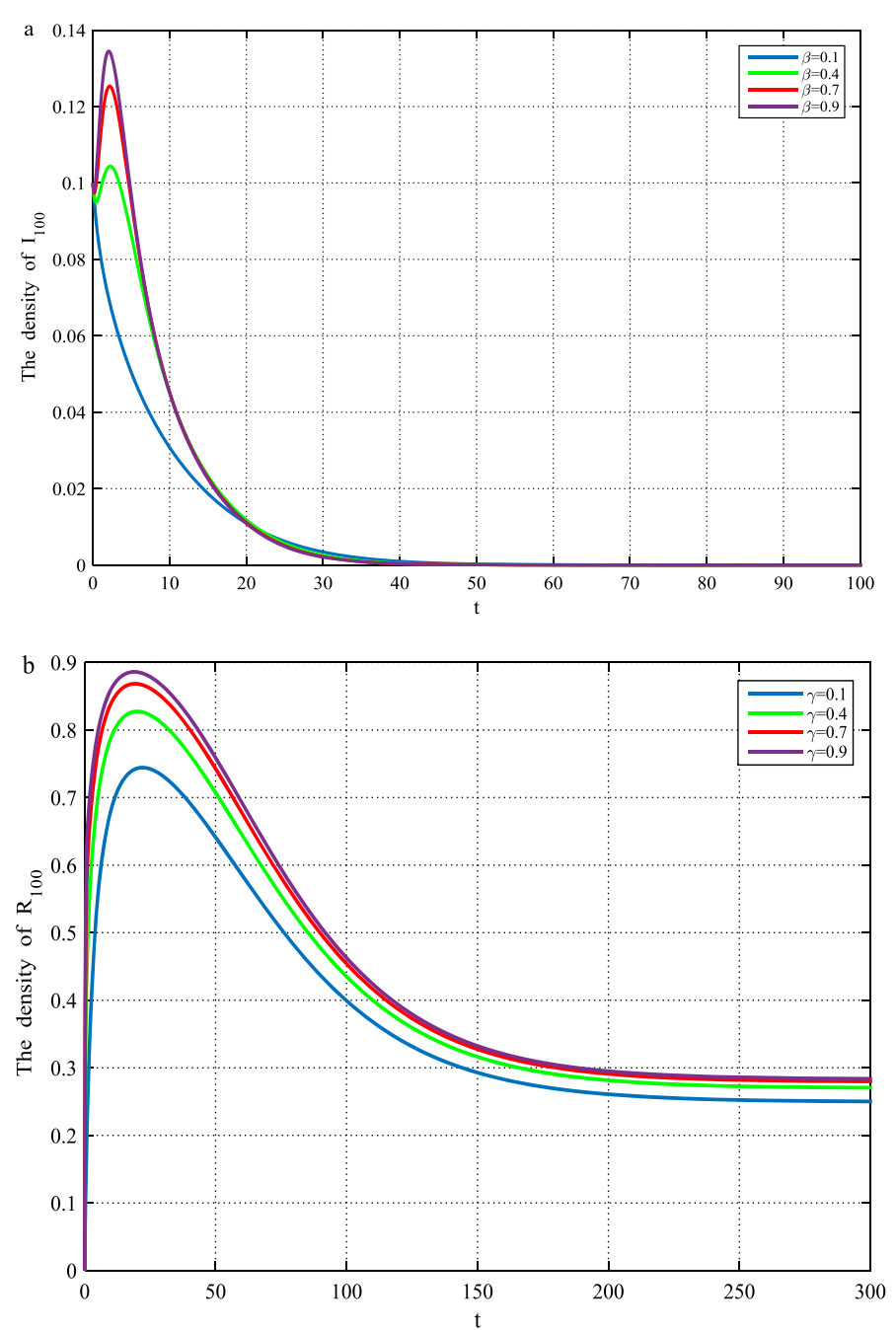

Figure 8 Density of $I_{100}$ versus $t$ varying over different infected rate $\beta$ in (a) and the density of $R_{100}$ versus $t$ varying over different direct immunization rate $\gamma$ in (b)

In Fig. 8(a), the prevalence $I_{100}$ versus $t$ corresponding to different infected rates $\beta$, which are chosen as $0.1,0.4,0.7,0.9$ from bottom to top, is shown. We can see that $I_{100}$ increases significantly as $\beta$ increases. In Fig. 8(b), the prevalence $R_{100}$ versus $t$ corresponding to different infected rates $\gamma$, which are chosen as $0.1,0.4,0.7,0.9$ from bottom to top, is illustrated. We can easily find that the density of the removed population increases as $\gamma$ increases.

In Fig. 9(a), the parameters are chosen as in Fig. 3(a) and the parameters in Fig. 5(b) are chosen as in Fig. 3(b). We can see that $I_{100}$ corresponding decreases significantly as the feedback parameter $\alpha$ increases, i.e., a larger feedback parameter can reduce the rumor spreading level.

\section{Conclusion}

This paper is mainly focused on the dynamics of rumor spreading on the complex social networks. A detailed SEIR rumor spreading model with hesitating mechanism has been presented and simulated. By using mean-field theory, we obtained the basic reproduction 

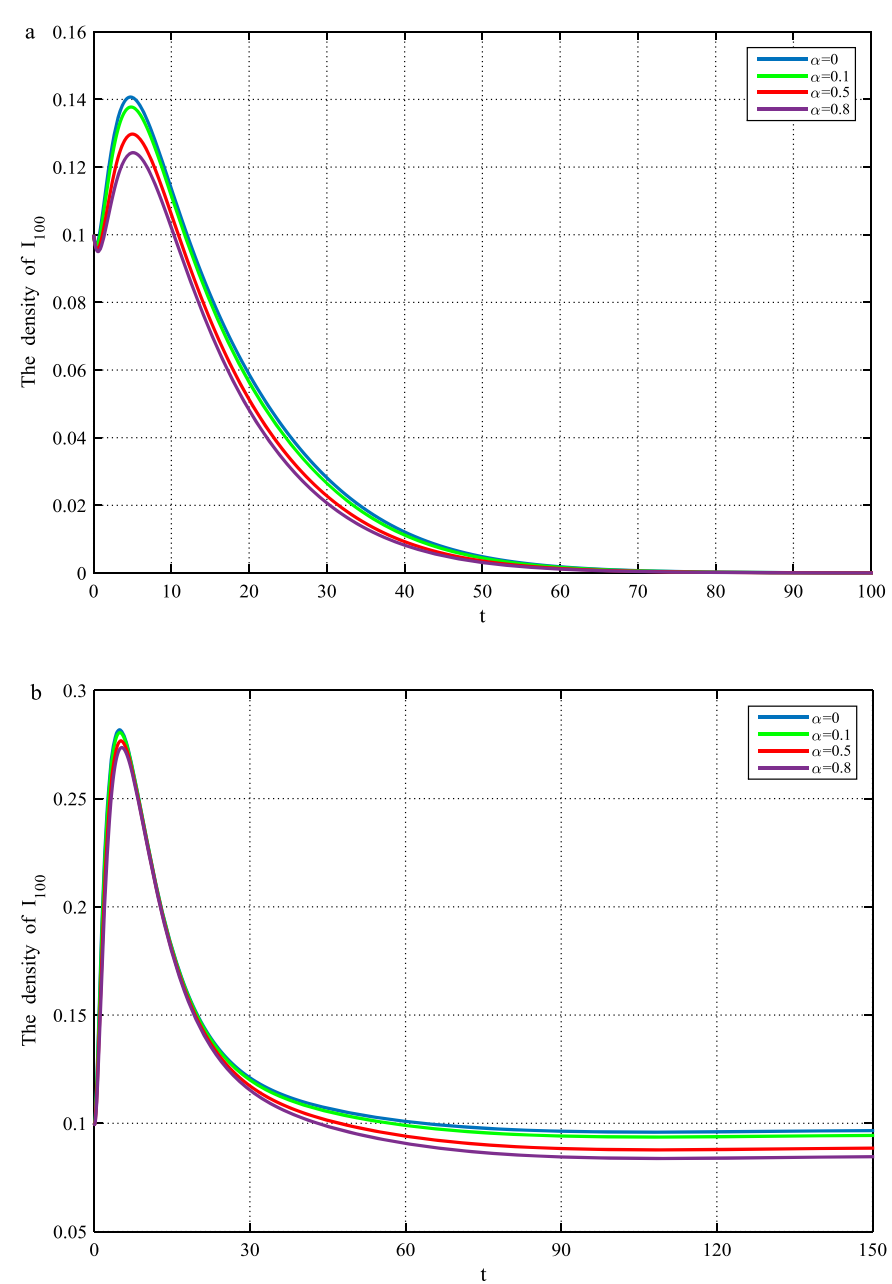

Figure 9 Density of $/_{100}$ versus $t$ with $R_{1}<1(\mathbf{a})$ and $R_{1}>1$ (b) to a different feedback parameter

number $R_{0}$ and the equilibrium. As the results indicate, the basic reproduction number in society networks is virtually correlated with the fluctuations of the degree distribution. Interestingly, $R_{0}$ bears no relation to the degree-dependent immigration $b(k)$. The global stability of equilibrium and the permanence have been proved in detail. We get the conclusion that higher attractiveness and fuzziness of rumor contribute to rumor spreading. Furthermore, increasing feedback parameters can result in the weakness of rumor spreading and the decrease of the population finally is infected. The study may give us valuable guidance to prevent the rumor spreading.

Acknowledgements

We thank the referees and the editor for their careful reading of the original manuscript and many valuable comments and suggestions that greatly improved the presentation of this paper.

Funding

This work is supported by the National Natural Science Foundation of China under Grants 61672112, 61873287 and Project in Hubei Province Department of Education under Grant B2016036. 
Authors' contributions

The authors contributed equally to this work. All authors read and approved the final manuscript.

\section{Author details}

${ }^{1}$ National Demonstration Center for Experimental Electrical and Electronic Education, Yangtze University, Jingzhou, P.R. China. ${ }^{2}$ School of Electronics and Information, Yangtze University, Jingzhou, P.R. China. ${ }^{3}$ School of Education Science, Tianjin Normal University, Tianjin, P.R. China.

\section{Publisher's Note}

Springer Nature remains neutral with regard to jurisdictional claims in published maps and institutional affiliations.

\section{Received: 14 May 2018 Accepted: 15 October 2018 Published online: 24 October 2018}

\section{References}

1. Galam, S.: Modeling rumors: the no plane pentagon French hoax case. Physica A 320, (2003) 571-580.

2. Bordia, P., Difonzo, N.: Psychological motivations in rumor spread, pp. 87-101 (2005)

3. Xu, J., Zhang, M., Ni, J.: A coupled model for government communication and rumor spreading in emergencies. Adv. Differ. Equ. 2016(1), 208 (2016)

4. Woo, J., Chen, H.: Epidemic model for information diffusion in web forums: experiments in marketing exchange and political dialog. SpringerPlus 5(1), 66 (2016)

5. Thomas, S.A.: Lies, damn lies, and rumors: an analysis of collective efficacy, rumors, and fear in the wake of Katrina. Sociol. Spectr. 27, 679-703 (2007)

6. Kesten, H., Sidoravicius, V.: The spreading of a rumor or infection in a moving population. Ann. Probab. 33, 2402-2462 (2005)

7. Wang, T., He, J., Wang, X.: An information spreading model based on online social networks. Physica A 490, 488-496 (2018)

8. Ren, F., Li, S.P., Liu, C.: Information spreading on mobile communication networks: a new model that incorporates human behaviors. Physica A 469, 334-341 (2017)

9. Daley, D.J., Kendall, D.G.: Stochastic rumours. IMA J. Appl. Math. 1, 42-55 (1965)

10. Maki, D.P., Thompson, M.: Mathematical Models and Applications. Prentice-Hall, Englewood Cliffs (1973)

11. Li, C.: A study on time-delay rumor propagation model with saturated control function. Adv. Differ. Equ. 1, 255 (2017)

12. Xia, C.Y., Ma, J.H.: Effect of distributed cure rate on the spreading behavior on complex networks. Energy Proc. 5 $1411-1415(2011)$

13. Nekovee, M., Moreno, Y., Bianconi, G., et al.: Theory of rumour spreading in complex social networks. Physica A 374(1), 457-470 (2007)

14. Roshani, F., Naimi, Y.: Effects of degree-biased transmission rate and nonlinear infectivity on rumor spreading in complex social networks. Phys. Rev. E 85(3), 036109 (2012)

15. Zhao, L., Wang, J., Chen, Y., et al.: SIHR rumor spreading model in social networks. Physica A 391(7), 2444-2453 (2012)

16. Xia, L.L., Jiang, G.P., Song, B., et al.: Rumor spreading model considering hesitating mechanism in complex social networks. Physica A 437, 295-303 (2015)

17. Pei, S., Muchnik, L., Tang, S.-T., Zheng, Z.-M., Makse, H.A.: Exploring the complex pattern of information spreading in online blog communities. PLOS ONE 10, e0126894 (2015)

18. Choudhary, A., Kumar, D., Singh, J.: A fractional model of fluid flow through porous media with mean capillary pressure. J. Assoc. Arab Univ. Basic Appl. Sci. 21, 59-63 (2016)

19. Huo, L., Wang, L., Song, N., et al.: Rumor spreading model considering the activity of spreaders in the homogeneous network. Physica A 468, 855-865 (2017)

20. Gu, J., Li, W., Cai, X.: The effect of the forget-remember mechanism on spreading. Eur. Phys. J. B 62, $247-255$ (2008)

21. Zhao, L., Qiu, X., Wang, X., Wang, J.: Rumor spreading model considering forgetting and remembering mechanisms in inhomogeneous networks. Physica A 392, 987-994 (2013)

22. Liu, C., Zhang, Z.K.: Information spreading on dynamic social networks. Commun. Nonlinear Sci. Numer. Simul. 19(4), 896-904 (2014)

23. Li, C., Ma, Z.: Dynamic analysis of a spatial diffusion rumor propagation model with delay. Adv. Differ. Equ. 1, 364 (2015)

24. Zhao, T., Hopf, B.D.: Bifurcation of a computer virus spreading model in the network with limited anti-virus ability. Adv. Differ. Equ. 1, 183 (2017)

25. Li, T., Wang, Y., Guan, Z.H.: Spreading dynamics of a SIQRS epidemic model on scale-free networks. Commun. Nonlinear Sci. Numer. Simul. 19,686-692 (2014)

26. Zhu, G., Fu, X., Chen, G.: Spreading dynamics and global stability of a generalized epidemic model on complex heterogeneous networks. Appl. Math. Model. 36, 5808-5817 (2012)

27. Zan, Y., Wu, J., et al.: SICR rumor spreading model in complex networks: counterattack and self-resistance. Physica A 405, 159-170 (2014)

28. Xia, L., Jiang, G.: Rumor spreading model considering hesitating mechanism in complex social network. Physica A 437, 295-303 (2015)

29. Wang, Y., Yang, X., et al.: Rumor spreading model with trust mechanism in complex social networks. Commun. Theor. Phys. 59, 510-516 (2013)

30. Li, P., Yang, X., Yang, L.X., et al.: The modeling and analysis of the word-of-mouth marketing. Physica A 493, 1-16 (2018)

31. Zhao, J., Wu, J., Feng, X., Xiong, H., Xu, K.: Information propagation in online social networks: a tie-strength perspective. Knowl. Inf. Syst. 32, 589-608 (2012)

32. Li, A.H., Wang, J.: Wang, Y.Q.: Research on CSER rumor spreading model in online social network. In: International Conference on Emerging Internetworking, Data \& Web Technologies, pp. 767-775. Springer, Cham (2017) 
33. Huo, L., Lin, T., Fan, C., et al.: Optimal control of a rumor propagation model with latent period in emergency event. Adv. Differ. Equ. 2015(1), 54 (2015)

34. Liu, Q., Li, T., Sun, M.: The analysis of an SEIR rumor propagation model on heterogeneous network. Physica A 469, 372-380 (2017)

35. Wan, C., Li, T., Sun, Z.: Global stability of a SEIR rumor spreading model with demographics on scale-free networks. Adv. Differ. Equ. 1, 253 (2017)

36. Escalante, R., Odehnal, M.: A deterministic mathematical model for the spread of two rumors (2017)

37. Zh, J., Sun, J.: Stability analysis of an SIS epidemic model with feedback mechanism on networks. Physica A 394, 24 (2014)

38. Li, T., Liu, X., Wu, J., et al.: An epidemic spreading model on adaptive scale-free networks with feedback mechanism. Physica A 450, 649-656 (2016)

39. Jinhuo, L., Wang, J., Wang, H.: Seasonal forcing and exponential threshold incidence in cholera dynamics. Discrete Contin. Dyn. Syst., Ser. B 22(6), 2261-2290 (2017)

40. Juher, D., Ripoll, J., Saldaña, J.: Outbreak analysis of an SIS epidemic model with rewiring. J. Math. Biol. 67(2), 411-432 (2013)

41. Ablikim, M., Achasov, M.N., Ai, X.C., et al.: Observation of a charged charmonium like structure in $e^{+} e^{-} \rightarrow \pi^{+} \pi^{-} J / \psi$ at $s=4.26 \mathrm{GeV}$. Phys. Rev. Lett. 110(25), 252001 (2013)

42. Boguñá, M., Pastorsatorras, R., Vespignani, A.: Epidemic spreading in complex networks with degree correlations. In: Statistical Mechanics of Complex Networks (2003)

43. Van den Driessche, P., Watmough, J.: Reproduction numbers and sub-threshold endemic equilibria for compartmental models of disease transmission. Math. Biosci. 180(1-2), 29-48 (2002)

44. Chen, F.: On a nonlinear nonautonomous predator-prey model with diffusion and distributed delay. Comput. Appl. Math. 180, 33-49 (2005)

45. Lajmanovich, A., Yorke, J.A.: A deterministic model for gonorrhea in a nonhomogenous population. Math. Biosci. 28, 221-236 (1976)

\section{Submit your manuscript to a SpringerOpen ${ }^{\circ}$ journal and benefit from:}

- Convenient online submission

- Rigorous peer review

- Open access: articles freely available online

- High visibility within the field

- Retaining the copyright to your article

Submit your next manuscript at $\gg$ springeropen.com 